https://helda.helsinki.fi

\title{
Analysis of infant cortical synchrony is constrained by the number of recording electrodes and the recording montage
}

\section{Tokariev, Anton}

2016-01

Tokariev , A , Vanhatalo , S \& Palva , J M 2016 , ' Analysis of infant cortical synchrony is constrained by the number of recording electrodes and the recording montage ' , Clinical Neurophysiology , vol. 127 , no. 1 , pp. 310-323 . https://doi.org/10.1016/j.clinph.2015.04.291

http://hdl.handle.net/10138/223862

https://doi.org/10.1016/j.clinph.2015.04.291

publishedVersion

Downloaded from Helda, University of Helsinki institutional repository.

This is an electronic reprint of the original article.

This reprint may differ from the original in pagination and typographic detail.

Please cite the original version. 


\title{
Analysis of infant cortical synchrony is constrained by the number of recording electrodes and the recording montage
}

\author{
Anton Tokariev ${ }^{\mathrm{a}, \mathrm{b}, *}$, Sampsa Vanhatalo ${ }^{\mathrm{b}}$, J. Matias Palva ${ }^{\mathrm{a}}$ \\ ${ }^{a}$ Neuroscience Center, University of Helsinki, Helsinki, Finland \\ ${ }^{\mathrm{b}}$ Department of Children's Clinical Neurophysiology, HUS Medical Imaging Center and Children's Hospital, Helsinki University Central Hospital and University of Helsinki, \\ Helsinki, Finland
}

\section{A R T I C L E I N F O}

\section{Article history:}

Accepted 24 April 2015

Available online 4 June 2015

\section{Keywords:}

Neonatal EEG

Forward model

Simulation

Dense array EEG

Connectivity

Brain development

\section{H I G H L I G H T S}

- Analysis of brain connectivity from the neonatal EEG is strongly enhanced by adding the number of electrodes.

- Sensitivity and specificity of cortical synchrony estimates depend on the analysis montage; average and Laplacian montage have the best performance.

- The number of electrodes defines the optimal montage and it also sets the limits for the level of analytic details.

\section{A B S T R A C T}

Objective: To assess how the recording montage in the neonatal EEG influences the detection of cortical source signals and their phase interactions.

Methods: Scalp EEG was simulated by forward modeling 20-200 simultaneously active sources covering the cortical surface of a realistic neonatal head model. We assessed systematically how the number of scalp electrodes (11-85), analysis montage, or the size of cortical sources affect the detection of cortical phase synchrony. Statistical metrics were developed for quantifying the resolution and reliability of the montages.

Results: The findings converge to show that an increase in the number of recording electrodes leads to a systematic improvement in the detection of true cortical phase synchrony. While there is always a ceiling effect with respect to discernible cortical details, we show that the average and Laplacian montages exhibit superior specificity and sensitivity as compared to other conventional montages.

Conclusions: Reliability in assessing true neonatal cortical synchrony is directly related to the choice of EEG recording and analysis configurations. Because of the high conductivity of the neonatal skull, the conventional neonatal EEG recordings are spatially far too sparse for pertinent studies, and this loss of information cannot be recovered by re-montaging during analysis.

Significance: Future neonatal EEG studies will need prospective planning of recording configuration to allow analysis of spatial details required by each study question. Our findings also advice about the level of details in brain synchrony that can be studied with existing datasets or by using conventional EEG recordings.

(c) 2015 International Federation of Clinical Neurophysiology. Published by Elsevier Ireland Ltd. All rights

reserved.

\footnotetext{
Abbreviations: 3D, three-dimensional; BEM, boundary element method; $\mathrm{CDH}$ cumulative density histograms; (c)DS, (cumulative) degree of smearing; (c)FNP, (cumulative) false negative probability; CSD, current source density; EEG, electroencephalography; hdEEG, high density EEG; IED, inter-electrode distance; $M$, number of discernible parcels; MEG, magnetoencephalography; MFC, montage fidelity coefficient; MRI, magnetic resonance imaging; PD, parcel diameter; $\mathrm{PDH}$, probability density histogram; PLV, phase locking value; (r/i)PLV, real/imaginary part of PLV.

* Corresponding author at: P.O. Box 56 (Viikinkaari 4), FI-00014, University of Helsinki, Finland. Tel.: +358 458788184

E-mail address: anton.tokariev@helsinki.fi (A. Tokariev).
}

\section{Introduction}

Interactions between brain areas are fundamental for most brain functions. These interactions may be observed with both invasive and non-invasive electrophysiological methods and appear to support neuronal communication, integration, and functional binding via spatiotemporal constellations of phase-correlated cortical oscillations (Stam and van Straaten, 2012). Several levels of evidence, ranging from simulations to 
experimental models and studies on human infants, support the idea that early neuronal network activities are crucial for the maturation of lifetime brain functions (Colonnese and Khazipov, 2012; Kilb et al., 2011). This has increased the interest in understanding how brain areas interact during early development (Grieve et al., 2008; Omidvarnia et al., 2014) and how the neurocognitive abnormalities arise from early adversities via altering early neuronal network activity (Krüger et al., 2012).

The introduction of novel dense array EEG recording methods (30-130 electrodes) into neonatal work has opened the possibility to study spatial details in neonatal brain activity in both hospital and laboratory settings (Grieve et al., 2008; Odabaee et al., 2013; Omidvarnia et al., 2014; Tokariev et al., 2012; Wallois et al., 2009). The recent theoretical work has shown that the information content, or spatial richness, of EEG signal that can be recorded from the neonatal scalp is dramatically higher than what is conventionally thought (Grieve et al., 2003, 2004; Odabaee et al., 2013). Pragmatically, this implies that conventional EEG recordings with eight to twenty electrodes (André et al., 2010) provide a significantly deficient representation of brain activity, because much if not most EEG activity available at scalp is ignored.

While the need for an increased number of recording electrodes has been established, there is no information about how the number of electrodes translates to the ability to study details of individual cortical activities, or how the neuronal interactions between brain areas are seen in the EEG when using different numbers of recording electrodes or analysis montages. Such information would be instrumental for several aspects of an appropriate study design on early brain network activity. First, it would be important to understand the level of neuroanatomical detail in brain function that can be plausibly studied using a limited number of scalp electrodes as is the case in most clinical recordings of sick infants. Second, it would be important to know how the recording montage affects the brain activity and interaction estimates, and how this depends on the number of recording electrodes. Third, defining the optimal recording and analysis settings, as well as estimating the trade-offs related to compromises, will be necessary in the scientific search of understanding early brain network function. While some of these issues have been studied in adults, the substantially different head geometry, much smaller dimensions and higher skull conductivity in infants lead to significant differences in the information yielded by EEG between infants and adults, and preclude the extrapolation of adult literature into the neonatal context. Notably, adult literature has focused on the effects of reference choice on amplitude-dependent measures of EEG (Essl and Rappelsberger, 1998; Nunez et al., 1997, 1999; Pascual-Marqui and Lehmann, 1993; Wolpaw and Wood, 1982; Yao et al., 2005, 2007), while the effects on phase synchrony have not been studied before.

\section{Methods and materials}

\subsection{Overview of the methodological approach}

We assessed here the accuracy with which local cortical dynamics and inter-areal interactions can be detected in human babies with variable numbers of scalp EEG electrodes and different re-referencing, i.e. montage options. This assessment was split into two parts: First, we used two complementary 'montage performance metrics' to index the performance of different montages and electrode numbers in detecting the cortical activity with variable parcellation resolutions. Second, we used two other 'coupling detection metrics' to see how well each montage and electrode number can detect coupling between signals from different cortical parcels, i.e. areas with coherent activity.
To quantify rigorously the relationship between scalp EEG signals and the underlying cortical (i.e. 'true' neuronal) signals, we obtained virtual EEG recordings by forward modeling simulated time series in cortical parcellations of variable resolutions (20200 parcels fully covering the cortical surface). As the basis for the forward model we used boundary element method (BEM; Geselowitz, 1967; Kybic et al., 2005) applied to 3D model based on an anatomical MRI of a healthy neonate.

An overview of the protocol is presented in Fig. 1, the generation of the head model is shown in Fig. 2, and the methods used to assess performance metrics for the different electrode numbers and montages are illustrated in Figs. 3 and 4.

\subsection{Head model generation}

Anatomical model: We used a magnetic resonance image (MRI, Philips 3T scanner, Helsinki University Central Hospital) of a healthy fullterm baby. Raw MRI slices (pixel size $0.9 \mathrm{~mm}$ ) were segmented manually (Fig. 1A, Fig. 2B) by a clinician using the FSL software (Jenkinson et al., 2012; Smith et al., 2004). 3D surfaces of the brain, inner skull, outer skull and scalp were reconstructed with Brainstorm software (Tadel et al., 2011). To improve computational performance, the scalp, inner skull, and outer skull surfaces were downsampled to 2562 vertices (5120 faces) and the brain surface to 4322 vertices ( 8640 faces). Fixed orientation dipoles normal to the vertices of the brain surface then comprised the source space with $\sim 3 \mathrm{~mm}$ separation between sources. Forward operator (Fig. 1B) for the resulting three-shell head model (Fig. 2C) was computed using symmetric boundary element method (BEM) in the OpenMEEG software (Gramfort et al., 2010; Kybic et al., 2005). To validate the results obtained with this source model as well as to examine the impact that a fully gyrated cortical surface would have on the output results, a 'gyrated model' was also used. In this model the original baby brain source model was replaced with a cortical surface source model with fixed surface-normal dipoles obtained from an adult subject, rescaled to the size of the baby brain and downsampled to have the same number of source vertices. The adult cortical surface (cortex-CSF border) was taken from 'Colin27' adult head model (Holmes et al., 1998) that is available in Brainstorm software.

Tissue conductivities: Following the recent study of Despotovic et al. (2013), we used the conductivity values $0.43 \mathrm{~S} / \mathrm{m}$ and $1.79 \mathrm{~S} / \mathrm{m}$ for the scalp and intracranial layer, respectively. Due to variability in the estimates of neonatal skull conductivities, we ran our simulations using two values, one that is near the traditional estimates $(0.033 \mathrm{~S} / \mathrm{m})$ and another that is closer to recent suggestions that neonatal skull layer is highly conductive $(0.2 \mathrm{~S} / \mathrm{m})$ (Despotovic et al., 2013; Grieve et al., 2004; Odabaee et al., 2013; Roche-Labarbe et al., 2008). In the gyrated model only skull conductivity of $0.2 \mathrm{~S} / \mathrm{m}$ was tested.

\subsection{Signal simulations}

\subsubsection{Simulation of cortical parcel signals}

To simulate cortical neuronal activity that is locally coherent, source dipoles oriented normally to the cortical surface were clustered (Figs. 1C and 2C) with closest neighbors using K-means approach (Baumgartner et al., 2000; Hanson et al., 2007) to give 20 to 200 cortical parcels $\left(N_{p}=20,40,60,80,100,120,140,160\right.$, 180 and 200). Increase in $N_{p}$ results in a decrease in the parcel size (see Fig. 2D for the parcel diameters) and the corresponding scalp potentials. We created a new cortical parcellation for each iteration of the simulations in order to eliminate the possibility of any given realization of the source parcellation introducing systematic distortions into the results. For each parcel, a unique parcel signal $S_{p}(t)$ was generated (white noise; nominal sampling frequency 


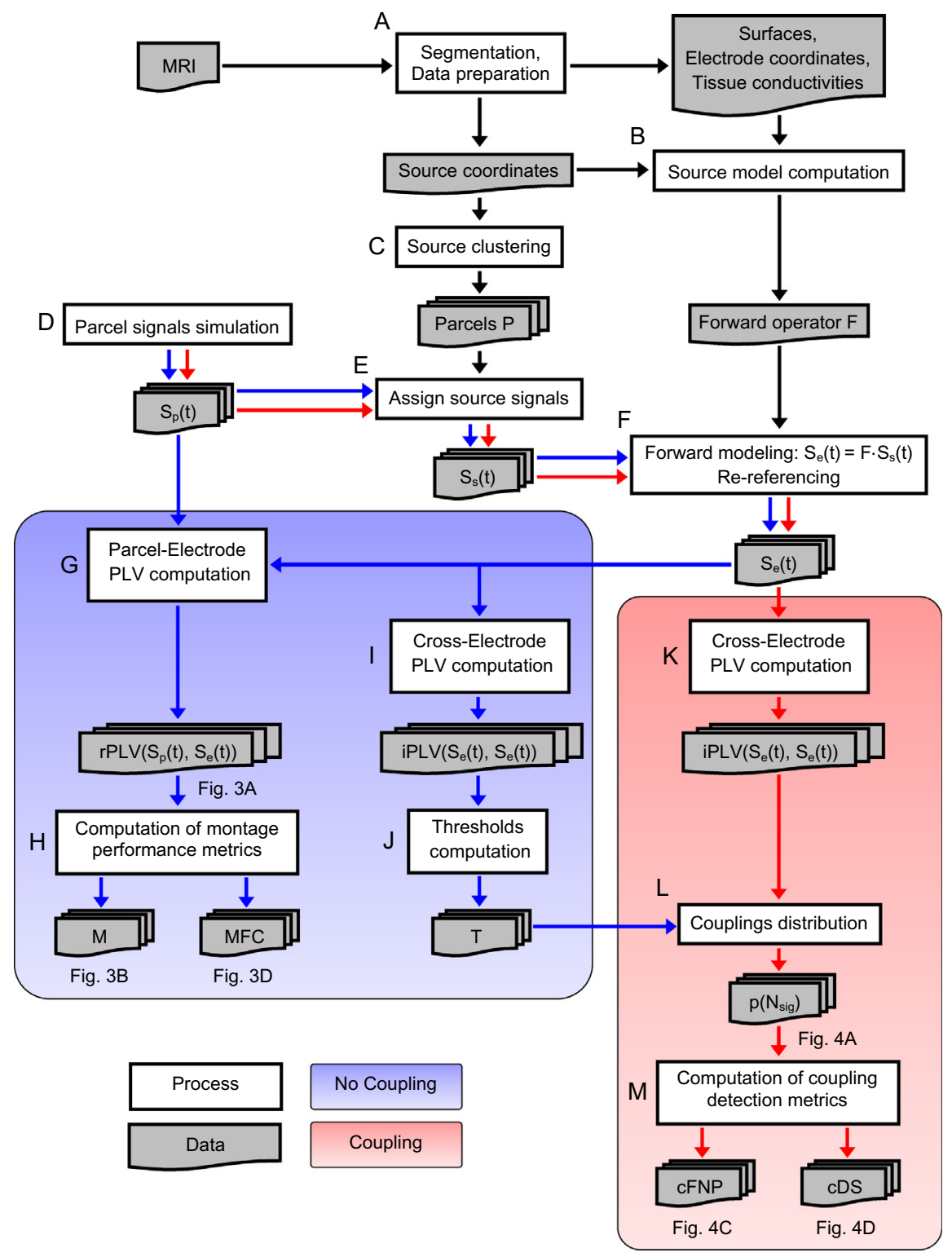

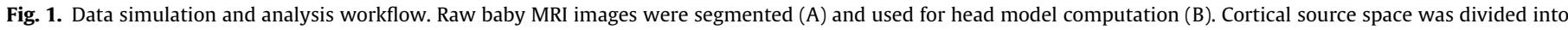

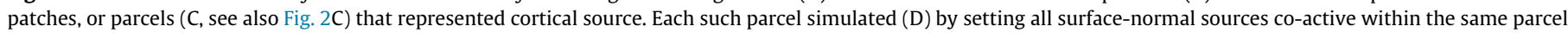

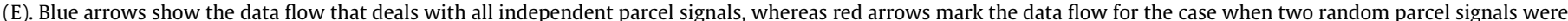

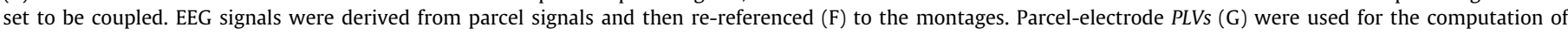

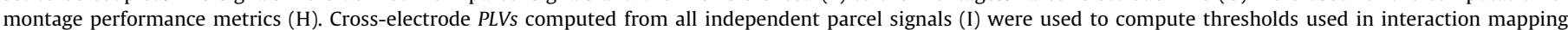

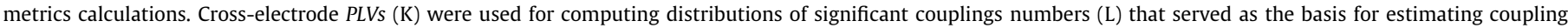

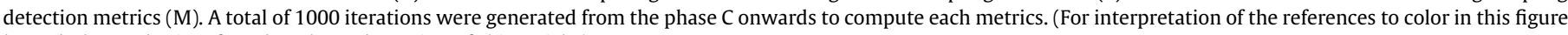
legend, the reader is referred to the web version of this article.)

$F s=100 \mathrm{~Hz}$, length 48000 samples; Fig. 1D) and convoluted with a Morlet wavelet:

$w\left(t, f_{0}\right)=A \cdot e^{-t^{2} / 2 \sigma_{t}^{2}} \cdot e^{2 j \pi f_{0} t}$,

where $A$ is a normalization factor, $f_{0}$ is a nominal frequency, $j$ is an imaginary unit and $\sigma_{t}=m^{\prime} / 2 \pi f_{0}$. We used the following settings: $f_{0}=10 \mathrm{~Hz}$ and $m^{\prime}=5$. Further, time series were downsampled with a factor of 48 to yield 1000 independent (separated by six wavelet time-domain standard deviations) complex-valued samples for later estimation of phase correlation. It should be noted that because all analyses in this study are focused on simulated narrow-band signals, neither the nature of noise (white or $1 / f$ ) nor the nominal wavelet frequency used in their generation play any significant role in the narrow-band amplitude or phase dynamics.
Simulations for estimating 'montage performance metrics' (pathway D-E-F-G-H on Fig. 1), were carried out by setting all $S_{p}(t)$ independent, while simulations for computing 'coupling detection metrics' (pathway D-E-F-K-L-M on Fig. 1) were done by imposing perfect phase coupling with $3 / 10$ cycle phase difference in one random pair of parcels while maintaining uncorrelated activity with equal amplitude in all other parcels (Fig. 2C). In all forward simulations, all source dipoles in each parcel were given identical time series to represent locally coherent cortical activity (Fig. 1E).

\subsubsection{Generation of scalp EEG signals and electrode sets}

Simulated cortical source signals $\left(S_{s}(t)\right)$ were transformed to scalp potentials $\left(S_{e}(t)\right)$ with a forward operator $F$ (Fig. 1F; Hämäläinen and Ilmoniemi, 1994; Lin et al., 2006): 
A

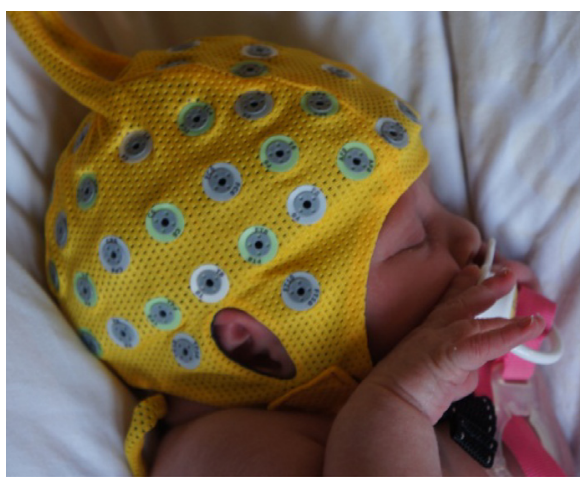

C

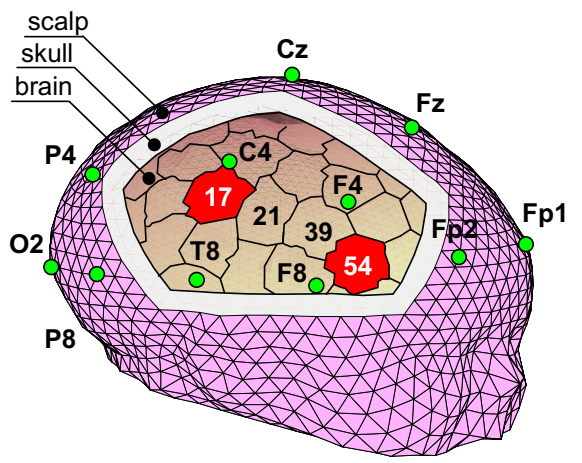

B

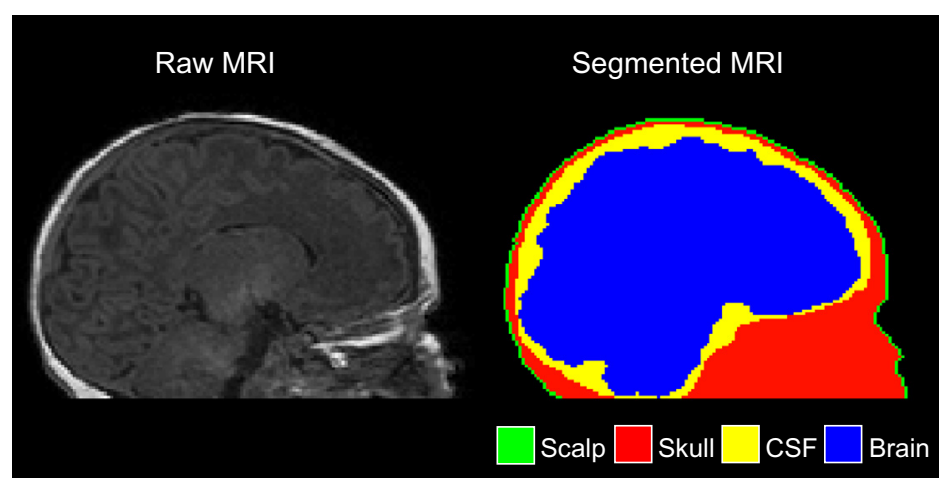

D

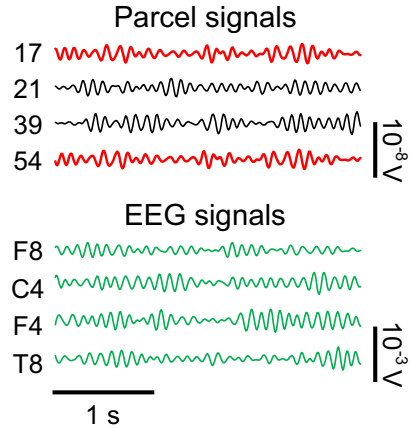

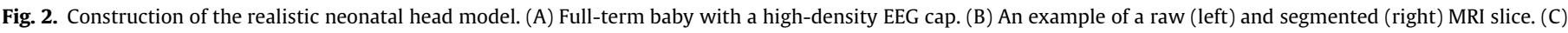

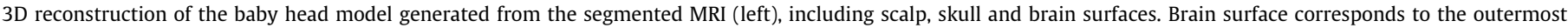

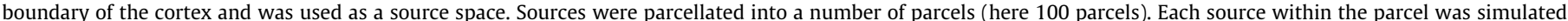

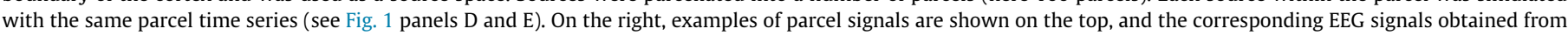

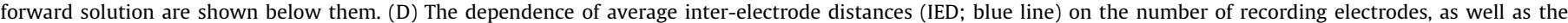
dependence of average parcel diameters (PD, red line) on the number of cortical parcels.

\section{$S_{e}(t)=F \cdot S_{s}(t)$}

We modeled up to 85 EEG electrodes placed on the scalp surface by using electrode coordinates from digitized 64-channel EEG cap on a doll head (Waveguard, ANT B.V., Enschede, The Netherlands, www.ant-neuro.com; see also Odabaee et al., 2013). The remaining 21 electrodes were placed manually on the model scalp according to the standard 10-10 system. The other electrode sets (with 66, 32,19 and 11 electrodes) were created as subsets of these 85 electrodes (Supplementary Figure S1). Notably, the higher electrode numbers $\left(N_{e}\right)$ are associated with both an improved spatial resolution and also a greater coverage of the scalp especially in the lower temporo-occipital region (for details, see Supplementary Material). Average interelectrode distances for each electrode set are shown in Fig. 2D. The scalp potentials were obtained with the forward operator in each electrode location in a Reference-free form that can be considered to be the least biased form of scalp EEG. Albeit available only in simulations, we used Reference-free EEG as a benchmark for the other montages available for real EEG recordings as well.

\subsubsection{Montaging}

Based on the Reference-free scalp potentials, we derived EEG using different sets of standard electrode locations (Supplementary Figure S1) comparable to actual EEG recordings with corresponding numbers of electrodes. Subsequently, these sets of EEG signals were used to compute the following data transformations (hereafter called montages) that are routinely used in the research and clinical practice. First, the average montage was calculated by subtracting the average of all electrode signals from each signal in the given electrode set. Second, the monopolar montage was computed by using $C z$ as the reference. Third, the current source density (CSD, also known as the surface Laplacian) montage was computed using spline spherical interpolation method as implemented in CSD Toolbox (Kayser and Tenke, 2006a,b; Tenke and Kayser, 2012; http://psychophysiology.cpmc.columbia.edu/ Software/CSDtoolbox; spline flexibility set to default value $m=4$ ) and can be seen as a scalp-level proxy for intracortical current density estimates (Tenke and Kayser, 2012). Since the CSD estimates are sensitive to the number of electrodes and a smoothing factor $(\lambda)$, we tested three levels of smoothing for each electrode set: (i) no smoothing $(\lambda=0, C S D)$, (ii) typical smoothing used in the implementation of e.g. BESA software (MEGIS GmbH, Gräfelfing, Germany; see Scherg et al., 2002) that depends on the number of scalp electrodes (see below, CSD default), as well as strong smoothing with the typical

(as in ii) smoothing factor multiplied by 10 (CSD strong). To the best of our knowledge, no studies have suggested optimal smoothing values for baby EEG. The following smoothing values are used in the adult EEG implementation of BESA software: $\lambda=10^{-5}$ for sets with 11,19 and 32 electrodes, and $\lambda=2 \cdot 10^{-6}$ for 66 and 85 electrodes. Fourth, common clinically used bipolar montages; banana, transverse, and linked mastoids montages were calculated from the conventional set of 19 electrodes placed according to the International 10-20 system. 
A
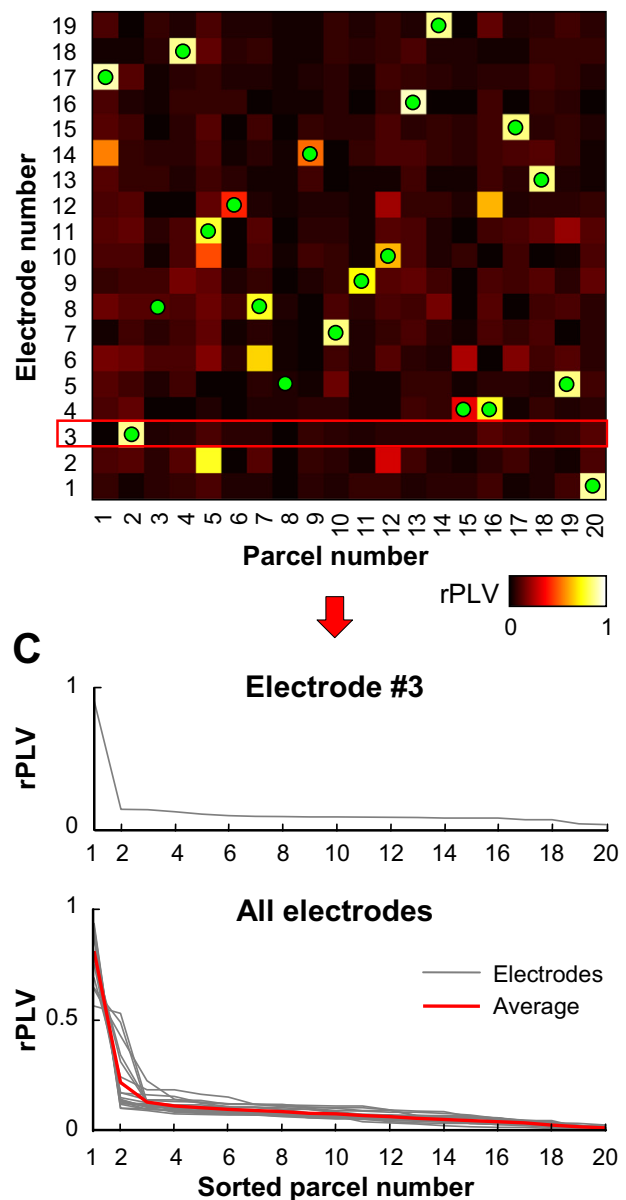

B

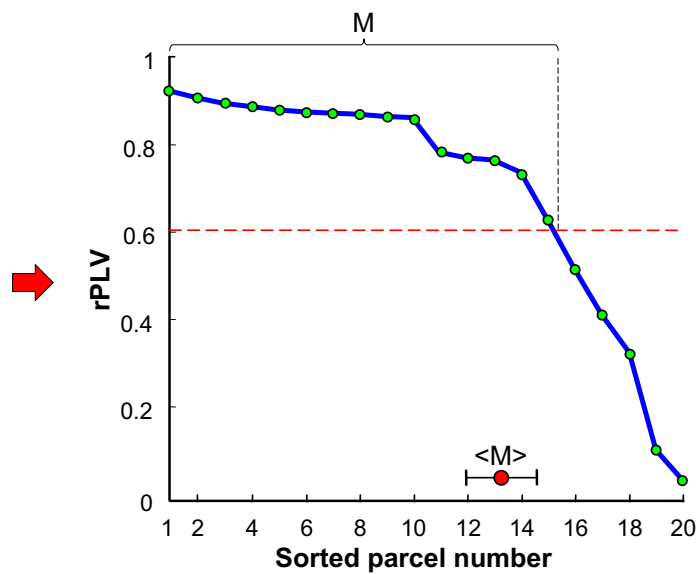

D

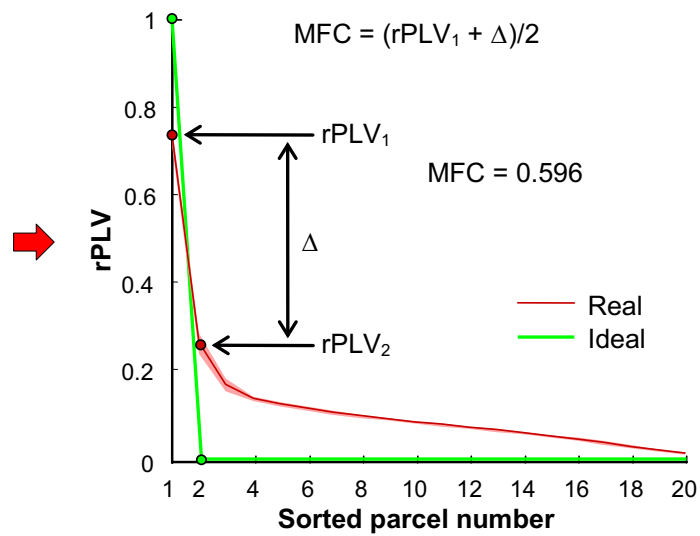

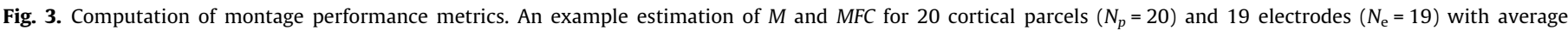

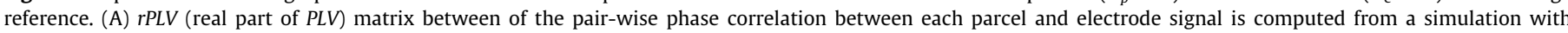

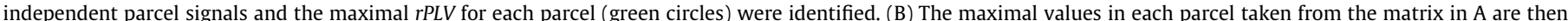

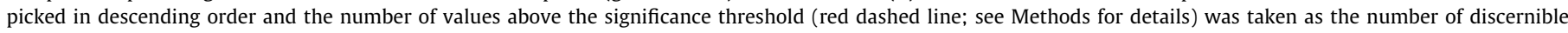

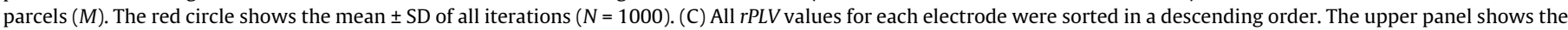

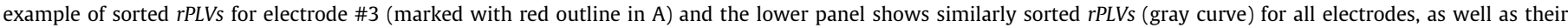

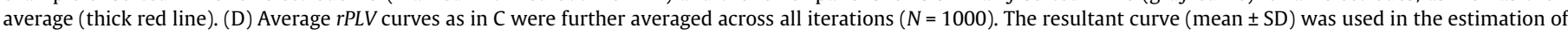

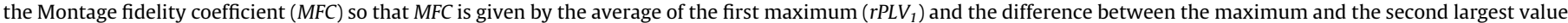
$\left(\Delta=r P L V_{1}-r P L V_{2}\right)$. The green line illustrates how an ideal montage would look in this assessment.

\subsection{Montage performance metrics}

The overall correlation of scalp EEG with the true underlying local cortical dynamics was evaluated with two approaches: the Number of discernible parcels $(M)$ and the Montage Fidelity Coefficient (MFC). Both measures were computed for all cortical parcellation resolutions $\left(N_{p}=20-200\right)$, all numbers of scalp channels $\left(N_{e}=11-85\right)$, and all montages. Both $M$ and MFC were extracted from estimates of phase correlation between cortical source (parcel) signals $S_{p}(t)$ and forward-modeled scalp EEG (electrode) signals $S_{e}(t)$ (Fig. 1G). As a synchrony measure, we used the real part $(r P L V)$ of the complex phase-locking value (PLV, Aydore et al., 2013; Jervis et al., 1983) because volume conduction and the mixing of uncorrelated signals from many sources at each electrode do not introduce systematic phase lags between the source and electrode signals.

$r P L V=\mid \operatorname{Re}\left\{\frac{1}{N} \sum_{k=1}^{N} e^{j \Delta \varphi(k)}\right\}$, where $\Delta \varphi$ denotes phase difference between two data series, $N$ is a time window length, $k$ is the sample number, $j$ is an imaginary unit and $|\cdot|$ indicates the absolute value.

PLV measures the uniformity of the distribution of $\Delta \varphi$ so that for uncorrelated phases and hence uniformly distributed $\Delta \varphi, P L V$ approaches 0 , when $N \rightarrow \infty$. For perfectly correlated phases and delta-function distributed $\Delta \varphi, P L V=1$.

Both $M$ and $M F C$ were estimated in 1000 iterations, each with 1000 independent samples, and the results are here reported as the averages of these iterations for each montage, $N_{p}$ and $N_{e}$.

\subsubsection{Number of discernible parcels ( $M)$}

From the $N_{p} \times N_{e}$ sized matrix of pair-wise rPLVs between all pairs of $S_{p}$ and $S_{e}$, maximal $r P L V$ s for each parcel signal versus all electrode signals were taken (Fig. 3A). $M$ was defined to be the number of $r P L V$ values exceeding a significance threshold (Fig. 3B). $M$ hence denotes the number of independent cortical parcels for each parcellation resolution with which the electrode signals of a given montage are highly correlated with. The threshold, 
A

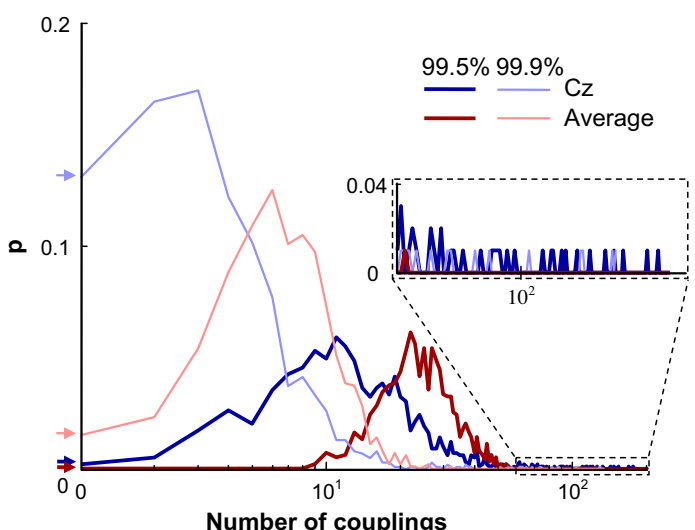

C

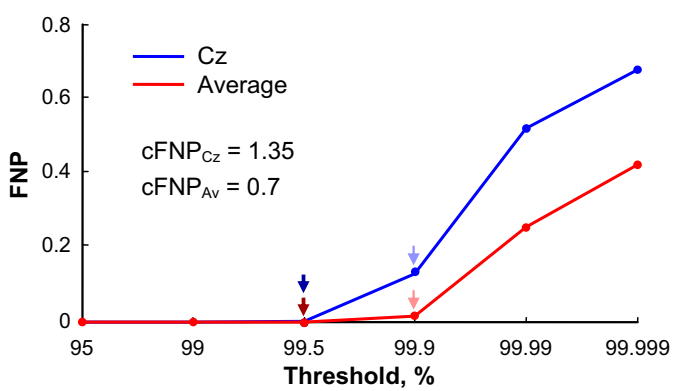

B

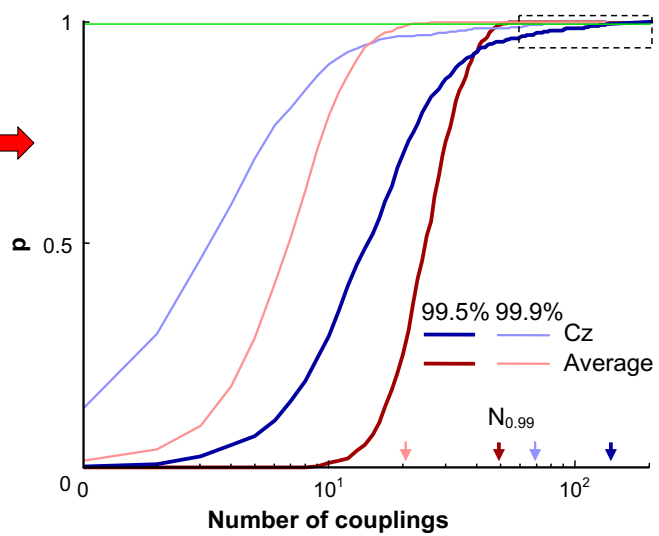

D

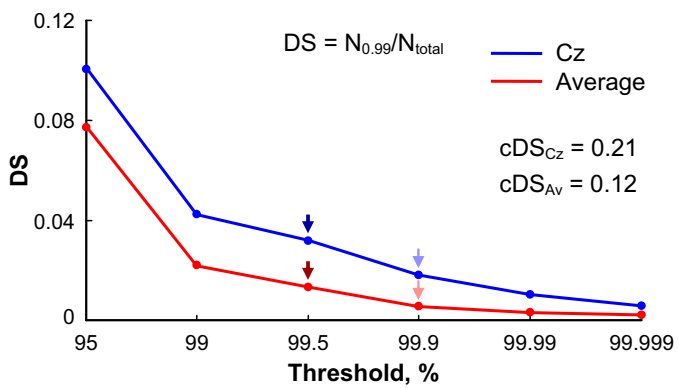

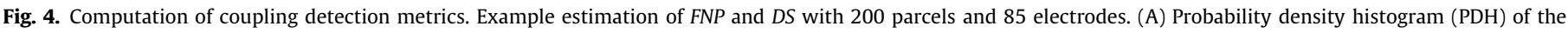

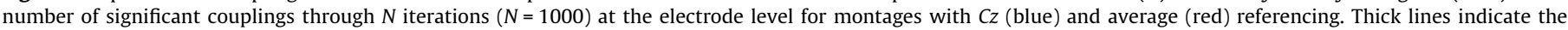

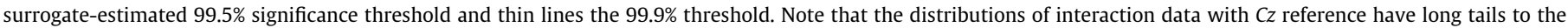

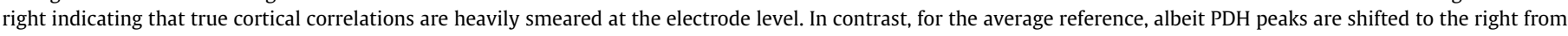

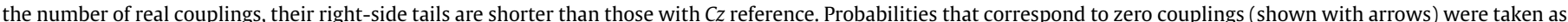

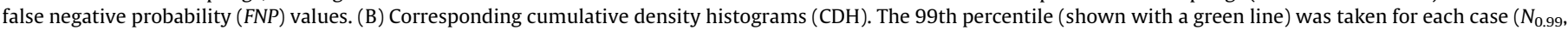

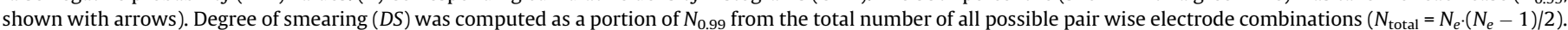

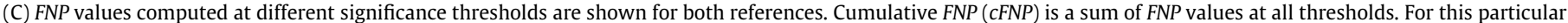

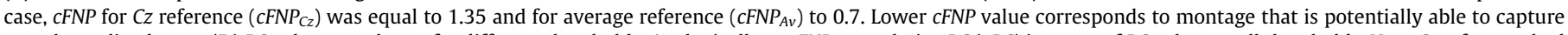

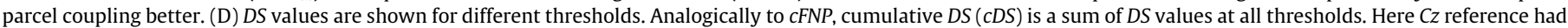
$c D S_{C z}=0.21$ and average reference $c D S_{A v}=0.12$. Lower $c D S$ value means that montage smears existing parcel coupling in a less degree.

0.61, was taken as a three standard deviations of all parcel-electrode $r P L V$ s computed for all montage and $N_{p}$ combinations (1000 iterations for each) and then kept fixed in all estimates of $M$. The threshold value was the same for the head models with different skull conductivities. We also tested thresholds of 0.5 and 0.7 and found that the relations between montages remained stable (Supplementary Figure S2).

\subsubsection{Montage fidelity coefficient (MFC)}

To compute MFC, rPLVs for each electrode versus all existing parcels in current parcellation were sorted in a descending order and averaged for all electrodes present in the set (for graphical explanation, Fig. 3C). Such average curves were computed in 1000 iterations and averaged again. We refer to resultant curve as to montage characteristic curve (Fig. 3D). MFC was calculated as a mean of the first maximal $r P L V\left(r P L V_{1}\right)$ and the difference between the first and the second maximal rPLVs $(\Delta)$ on the montage characteristic curve:

$M F C=\frac{r P L V_{1}+\Delta}{2}$,

The metric shows how well the electrodes in certain montage are 'focused' on as few as possible cortical parcels as opposed to picking up the mixed signal from many parcels. In the theoretical case of an 'ideal' montage (green characteristic curve in Fig. 3D) $r P L V_{1}$ would be 1 , which indicates that each electrode is perfectly sensitive to a single parcel signal, and $\Delta$ would be 1 indicating that no correlations with other parcels take place (perfect specificity). MFC hence quantifies the extent to which volume conduction and anatomical proximity of the cortical sources limit the real-life montage fidelity.

\subsection{Coupling detection metrics}

The ability of scalp level electrodes to measure cortical level coupling was assessed with coupling detection metrics that were designed to evaluate the two key challenges. First, a scalp recording may miss true parcel coupling because of incomplete scalp electrode coverage relative to the location or anatomical extent of cortical activities. We used false negative probability (FNP) to assess how sensitive the given scalp recording is in detecting cortical coupling. Second, cortical coupling is smeared at the scalp level because of volume conduction and mixing of signals from neighboring sources (Tognoli and Kelso, 2009) and hence each one correlated pair of cortical areas will be picked up as correlations between many scalp electrodes. The susceptibility of a given montage to signal mixing was estimated by Degree of smearing (DS). 
To quantify the phase correlations between two electrode time series, we used as an interaction metric the imaginary PLV (iPLV; Vinck et al., 2011) that reveals true phase-lagged interactions and is insensitive to the direct (zero-phase lag) effects linear mixing and volume conduction (or lead field overlap) at the expense of also being insensitive to true zero-phase lag neuronal interactions:

$i P L V=\left|\operatorname{Im}\left\{\frac{1}{N} \sum_{k=1}^{N} e^{j \Delta \varphi(k)}\right\}\right|$

\subsubsection{Computation of thresholds}

We estimated the statistical iPLV thresholds in the nullhypothesis condition so that independent parcel time series were simulated, forward modeled, and then all-to-all cross-electrode iPLVs were computed for 1000 iterations, for each combination of $N_{e}$ versus $N_{p}$, and for all montages (Fig. 1I). The size of this surrogate set was about $4 \cdot 10^{8} i P L V$ s. Significance thresholds were computed as $n$ percentiles of the surrogate set (Fig. $1 \mathrm{~J}$ ). We used $n=0.95$, $0.99,0.995,0.999,0.9999$ and 0.99999 in coupling detection analyses (corresponding to iPLVs 0.042, 0.056, 0.061, 0.071, 0.085 and 0.096 respectively). Multiple thresholds were used to corroborate the robustness of the findings. Values of the thresholds obtained with both head models were essentially the same.

\subsubsection{False negative probability (FNP)}

First, all pairwise inter-electrode $i P L V s$ were calculated (Fig. $1 \mathrm{~K}$ ). Second, the numbers $i P L V$ s exceeding each given significance threshold were counted $\left(N_{\text {sig }}\right)$. This was repeated for 1000 iterations with a new cortical parcellation and new inter-parcel connectivity matrix in each iteration. These 1000 numbers of significant couplings were pooled to a probability density histogram (PDH; Fig. 1L, Fig. $4 \mathrm{~A})$. The first bin of PDH $\left(N_{\text {sig }}=0\right)$ was taken as FNP. This metric shows the probability of missing existing parcel coupling by montage. Lower FNP value means that the montage catches brain signal couplings more reliably. Such FNP values were calculated at different significance thresholds (examples of FNP curves are shown on Fig. 4C). As far as FNP curves for different references at fixed $N_{p}$ and $N_{e}$ were separable, we used cumulative FNP (cFNP; sum of FNP values at all thresholds) to characterize and compare montages:

$c F N P=\sum_{i=1}^{n} F N P_{i}$,

where $n$ is an overall number of tested significance thresholds and $i$ its current number.

\subsubsection{Degree of smearing (DS)}

$D S$ was estimated from the data containing random-pairwise interactions, and it was computed from the cumulative density histogram (CDH; Fig. 4B) of the corresponding PDH (Fig. 4A). DS was taken as the ratio of couplings exceeding the 99th percentile of surrogate data $\left(N_{0.99}\right)$ to the total number of possible pairwise electrode combinations in a montage ( $N_{\text {total }}$; see also Fig. $4 \mathrm{D}$ for examples):

$D S=N_{0.99} / N_{\text {total }}$,

whereas, $N_{\text {total }}=N_{e} \cdot\left(N_{e}-1\right) / 2$.

Like FNP (see above), DS was estimated at the same significance thresholds. In the analyses, we used cumulative $D S(c D S)$ to obtain a robust and threshold-independent measure of $D S$ :

$c D S=\sum_{i=1}^{n} D S_{i}$

where $n$ is an overall number of tested significance thresholds and $i$ its current number. Low values of $c D S$ indicate that the linear mixing characteristic to a given montage smears true parcel-parcel coupling less than the mixing in a montage with high $c D S$.

\section{Results}

3.1. Analyses of EEG signal sensitivity with the number of discernible parcels, $M$

To estimate how well EEG electrode signals represent only a few well delineated underlying sources as opposed to yielding mixtures of many underlying sources, we introduce here the $M$ analysis where $M$ denotes the number of independent cortical parcels with which the electrode signals are highly correlated with. Exploring the $M$ values for different montages, parcellation resolutions, and EEG electrode numbers, we found $M$ to strongly depend on the relationship between inter-electrode distance (IED) and the diameter of cortical parcel (PD, respectively; Fig. 2D). When the IED is less than $\mathrm{PD}$, signals from most if not all cortical parcels may be picked up by electrodes at scalp (i.e. when $M$ approaches the number of simulated parcels $\left(N_{p}\right)$ see Fig. 5A and Supplementary Figure S3). Conversely, IED greater than the PD leads to rapid decrease in $M$. The maximum $M$ with each number of recording electrodes (marked with green circles on Fig. 5A) was found when IED and PD were roughly equal. When the resolution of the cortical parcellation increases to $>100$ parcels, however, the parcels become so small that the $M$ decreases irrespective of the number of electrodes $\left(N_{e}\right)$. This indicates that there is a clear upper limit to the level of neuroanatomical detail in cortical activity that is accessible with scalp EEG.

Increases of the skull conductivity is known to lead to higher spatial resolution in scalp potentials. We studied here conductivities $0.033 \mathrm{~S} / \mathrm{m}$ and $0.2 \mathrm{~S} / \mathrm{m}$ and found, expectedly, that the higher skull conductivity was associated with a greater $M$ albeit the overall patterns of $M$ as a function of montage, number of electrodes $\left(N_{e}\right)$, and number of parcels $\left(N_{p}\right)$ were similar (Fig. 5A). Among all tested montages, CSD without smoothing ('CSD') was least affected by the change in skull conductivity and yielded the greatest $M$. Comparison against the Reference-free (benchmark) montage showed that the CSD montage without smoothing introduced significant spatial filtering that made it spatially even more selective than the Reference-free and average montages that were mutually comparable (Fig. 5A).

Taken together, increasing the number of electrodes improved spatial precision and the ability to track multiple brain signals as indexed by $M$. $M$ values also depended on the choice of reference with $\operatorname{CSD}\left(\lambda=0, N_{e}=85\right)$ yielding best $M$ values at all $N_{p}$ for recordings with 66 or 85 electrodes. However, with 32 or less electrodes, Reference-free and average montages were better.

\subsection{Quantification of EEG signal specificity with the montage fidelity coefficient, MFC}

To complement the EEG sensitivity estimation performed with $M$, we developed a measure, montage fidelity coefficient (MFC) to address the specificity of the electrode signals. MFC was estimated by quantifying for each electrode signal the correlation with each parcel time series and then measuring the ratio of the strongest and the second strongest correlation and averaging these ratios across electrodes (Fig. 3C, D). We found MFC to be largely determined by the resolution of the cortical parcellation so that an increasing number of parcels $\left(N_{p}\right)$ and thereby the increasing degree of signal mixing was paralleled by decreasing MFC in all tested montages (Fig. 5B). For electrode numbers < 66, average and Reference-free montages yielded superior MFC values whereas with 66 or 85 electrodes and cortical parcellations with 60 or more parcels, the CSD montage had the greatest specificity as indexed by $M F C$. 
A
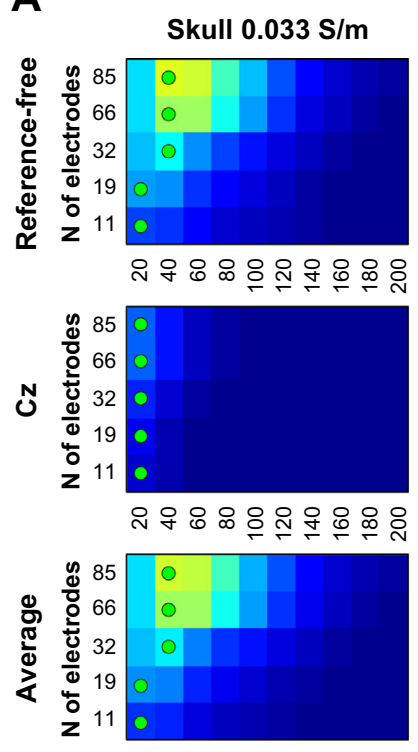

웡ㅇㅇㅇㅇㅇㅇㅇㅇㅇㅇㅇㅇㅇㅇ
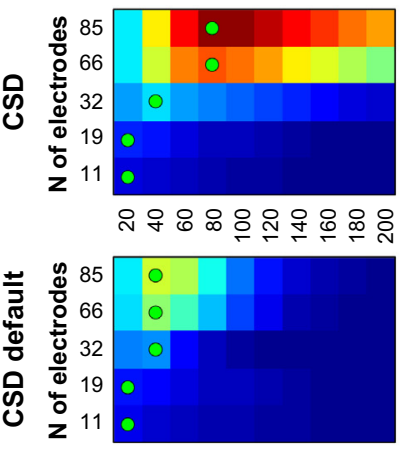

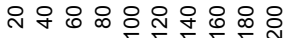

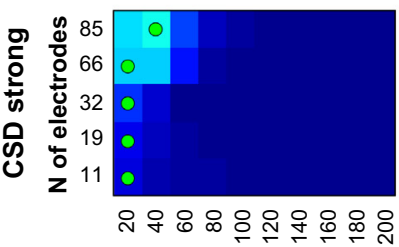

$\mathrm{N}$ of parcels
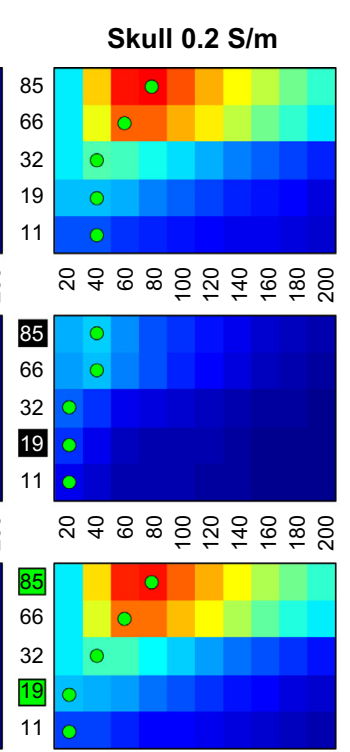

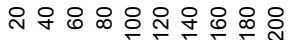
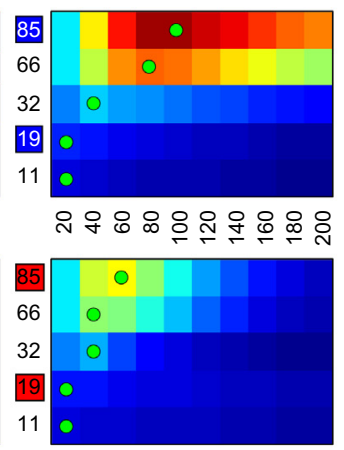

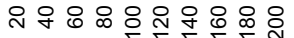

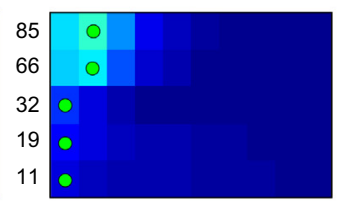

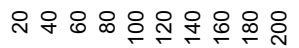

$\mathrm{N}$ of parcels
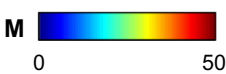

B
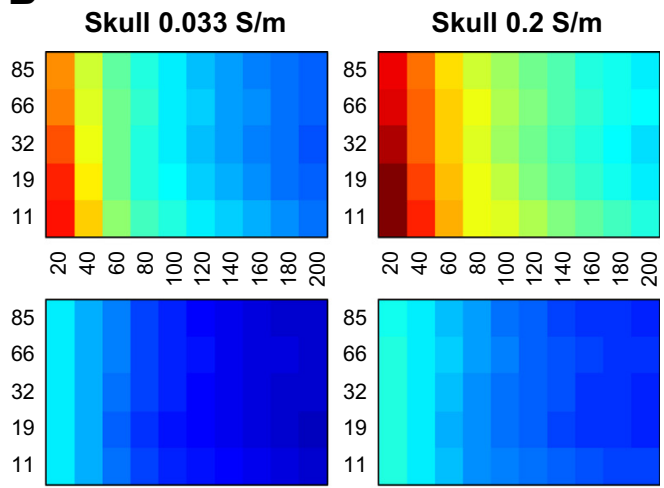

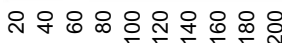

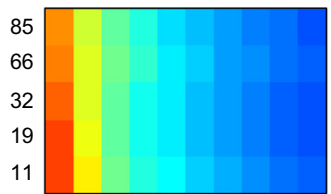

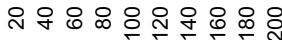

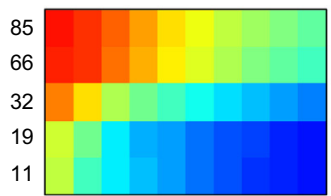

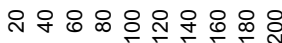

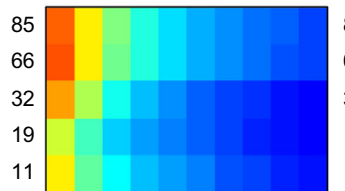

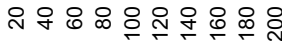
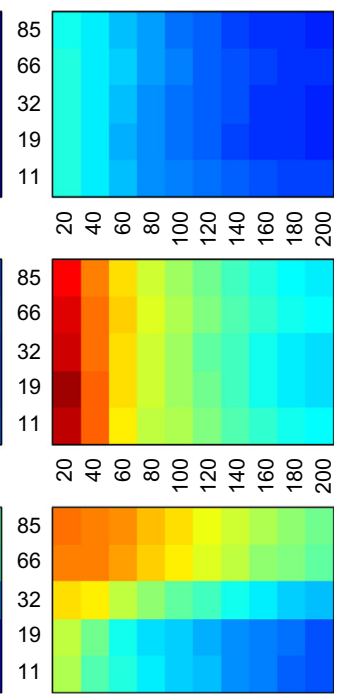

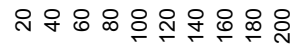

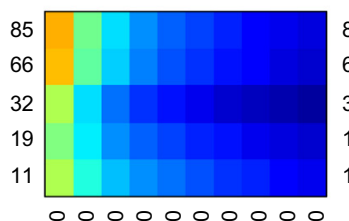

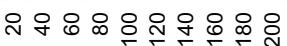

$\mathrm{N}$ of parcels

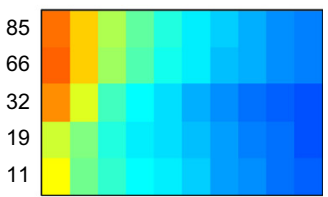

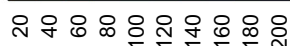

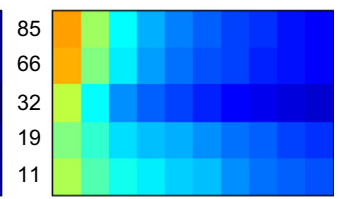

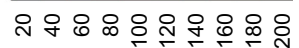

$\mathrm{N}$ of parcels

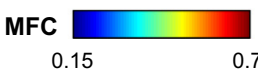

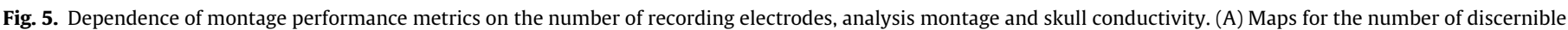

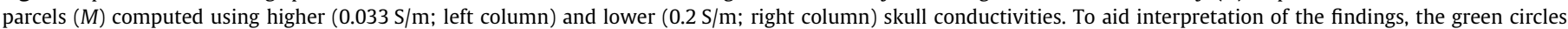

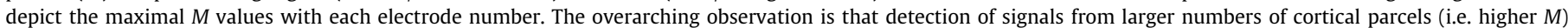

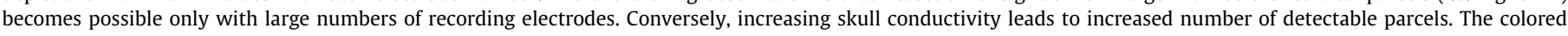

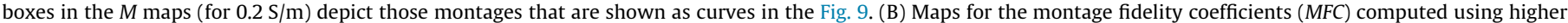
$(0.033 \mathrm{~S} / \mathrm{m}$; left column) and lower $(0.2 \mathrm{~S} / \mathrm{m}$; right column $)$ skull conductivities.

Skull conductivity had a clear effect on MFC (Supplementary Figure S4). With a lower skull conductivity $(0.033 \mathrm{~S} / \mathrm{m})$, the MFC values for Reference-free, average and $C z$ montages were smaller than those observed using higher conductivity $(0.2 \mathrm{~S} / \mathrm{m})$. On the other hand, $\operatorname{CSD}\left(\lambda=0, N_{e}=66,85\right)$ with $N_{p} \leqslant 60$, model with lower skull conductivity was associated with greater MFC and with $N_{p}>60$, the skull conductivity had little effect on MFC.

\subsection{Estimation of interaction mapping (in)sensitivity with the cumulative false negative probability (cFNP)}

The analyses of $M$ and MFC quantified how accurately EEG electrode signals capture the underlying local cortical dynamics. To estimate how informative these electrode signals are in the measurement of inter-areal cortical interactions, we used the $c F N P$ that measures the fraction of cortical interactions that a given montage fails to observe. cFNP hence parallels $M$ in yielding an (inverse) measure of sensitivity in interaction mapping. Unsurprisingly, with all montages, $c F N P$ increased with increasing $N_{p}$ (and decreasing parcel size, left column of Fig. 6). This increase was more prominent with low electrode counts. Both skull conductivities yielded similar results (Supplementary Figure S5). These trends indicate that in identification of neuronal interactions, it is always beneficial to record EEG with as high as possible density of electrodes. Higher electrode numbers will also allow increase in accuracy via using source level analysis. For highest densities of EEG tested in 
our study ( $N_{e}=66$ or 85 ), CSD without smoothing gave the lowest cFNP values and hence the best performance across all parcellation resolutions. With low to medium numbers of electrodes, $\left(N_{e}=11\right.$, 19 or 32 ), the best performance was, again, obtained with Reference-free and average montages.

\subsection{Quantification of the effects of volume-conduction related signal} mixing with cumulative degree of smearing ( $C D S)$

To complement the local specificity estimates (MFC) at the network level comparisons of montages, we approximated the volume-conduction related smearing with $c D S, c D S$ is a threshold-independent measure of the fraction of significant interactions and is, inversely like $c F N P$, related to goodness of the montage so that 'good' montages are expected to spread the discovered true cortical interactions to a smaller number of electrode-electrode couplings than 'poor' montages.

Expectedly, $c D S$ decreased with increasing parcellation resolution $\left(N_{p}\right)$ for all montages (Fig. 6, middle column). Absolute number of electrode pairs showing significant synchronization rose when the $N_{e}$ increased (Fig. 6, right column), however this was paralleled with a decrease in $c D S$
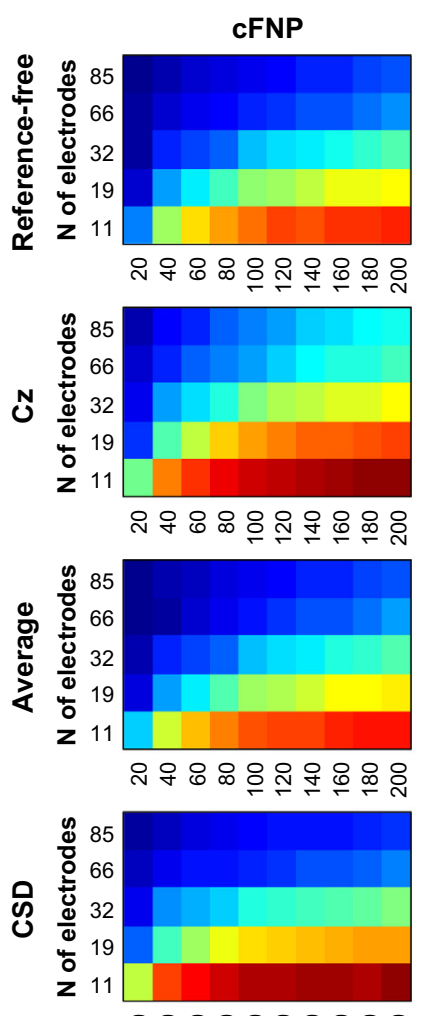

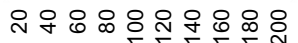

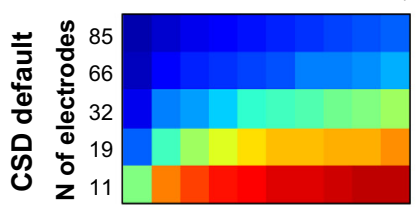

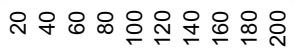
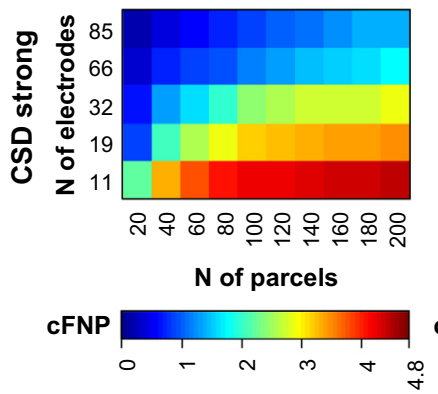

cDS

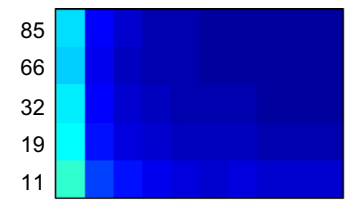

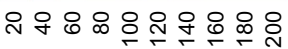
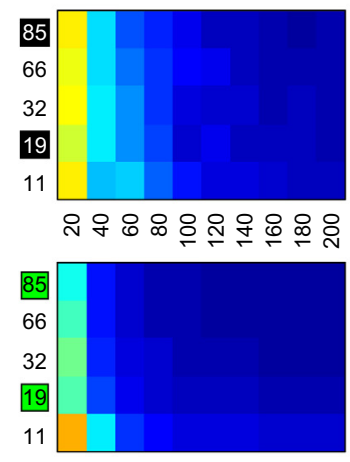

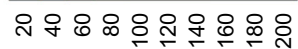
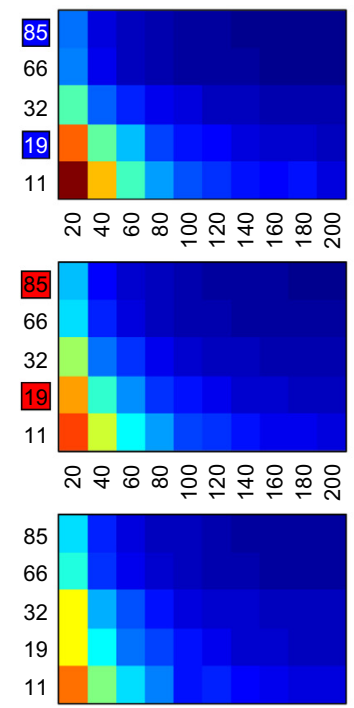

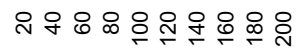

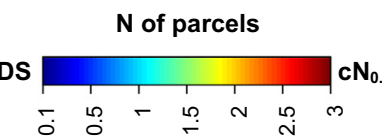

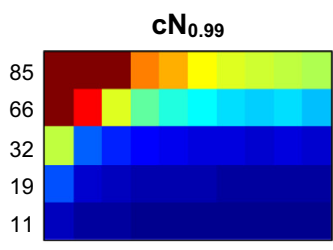

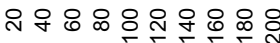
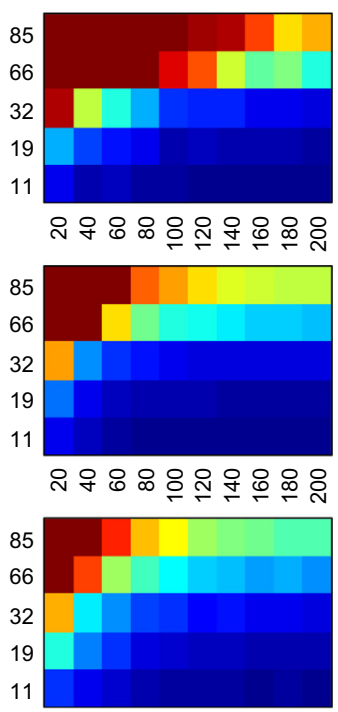

웅ㅇㅇ응ㅇㅁㅇㅇㅇㅇㅠ

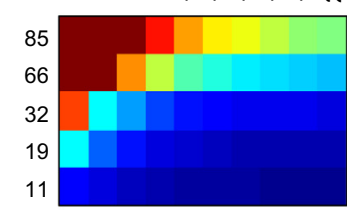

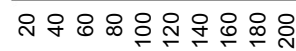

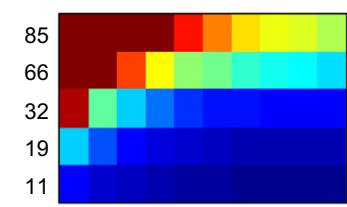

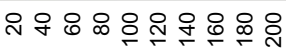

\section{$\mathrm{N}$ of parcels}

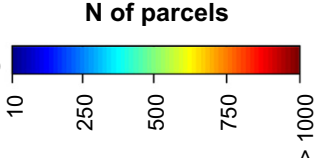

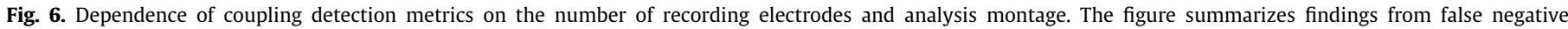

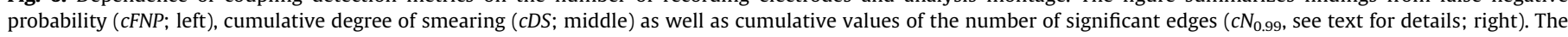

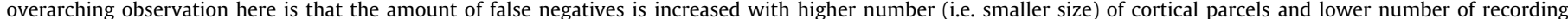

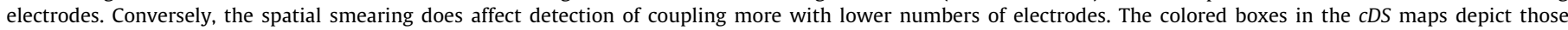
montages that are shown as curves in the Fig. 9. 
values. The overall pattern of $c D S$ findings was not affected by skull conductivity.

An overarching observation from all pairs of $N_{e}$ and $N_{p}$ was that the montage has a crucial effect on the accuracy of estimates of neuronal interactions. The higher density EEG recording $\left(N_{e}=66\right.$, $85)$ was expectedly most accurate with the un-smoothed $(\lambda=0)$ CSD montage that showed the smallest $c D S$ values even with the coarsest parcellations (large parcels and low $N_{p}$, see also Supplementary Figure S6). When the electrode density was decreased $\left(N_{e}=19-32\right)$, Reference-free and average montages appeared to be better than any CSD option. Taken together findings of all metrics ( $c F N P, C D S, M$ and $M F C$ ), it appears that the ability of a given montage to detect a cortical interaction depends both on the sensitivity in picking up the cortical signals and specificity with which they are extracted.

\subsection{Gyrated source model yields qualitatively and quantitatively similar results}

To corroborate the observations made with the baby cortex source model, we also used a fully gyrated surface source model (Fig. 7A) obtained from an adult subject and rescaled to the baby brain size. In the gyrated model $M$ values (Fig. 7B, left column) were lower (35\% lower with the CSD montage) compared to the original model (Fig. 5A, right column). This is expectable because deep sulcal sources give overall weaker signals and some sulcal parcels may exhibit partial signal cancelation by having sources with opposite polarities, and also because greater dipole orientation diversity and the dipolar scalp field potentials from sulcal sources are inherently associated with a greater extent of field spread. Accordingly, smearing $(c D S)$ for the gyrated model (Fig. 7B, right column) was somewhat greater ( $2 \%$ greater with the CSD montage) than with the original model (Fig. 6, middle column). However, the relationships between different montages as well as $M$ and $c D S$ behavior through different $N_{e}$ vs. $N_{p}$ combinations remain the same as in original baby model, which confirms that the choice of the source model per se does not grossly distort the results found in this study.

\subsection{Bipolar montages}

In clinical practice, small electrode numbers (e.g. $N_{e}=19$ ) and bipolar montages are routinely used (Fig. 8A). We assessed the performance of standard banana, transverse, and linked mastoids montages. The overall best performance was given by the linked mastoids reference that yielded higher values of $M$ and $M F C$ (Fig. 8B) and lower values of $c F N P$ than the banana and transverse montages (Fig. 8C). cDS values, on the other hand, were slightly better for banana and transverse (especially for $N_{p}<60$ ). Comparison of the linked mastoids montage at $N_{e}=19$ electrodes showed that only the average montage gave systematically better results. In the light of each performance metric used in this study, the 'monopolar' montage with $C z$ reference was found to yield poorest results.

\section{Discussion}

Our study shows that the neonatal scalp EEG can be effectively used to study cortical synchrony. Synchrony estimates are, however, markedly affected by the number of recording electrodes as well as by the analysis montage. In addition, the size of interacting cortical areas and the conductivity of skull tissue both have a notable effect on the assessment of synchrony. These findings are qualitatively compatible with the prior work on adult EEG simulations (Ryynanen et al., 2004, 2006) but our work extends the prior
A

A Original model

Gyrated model
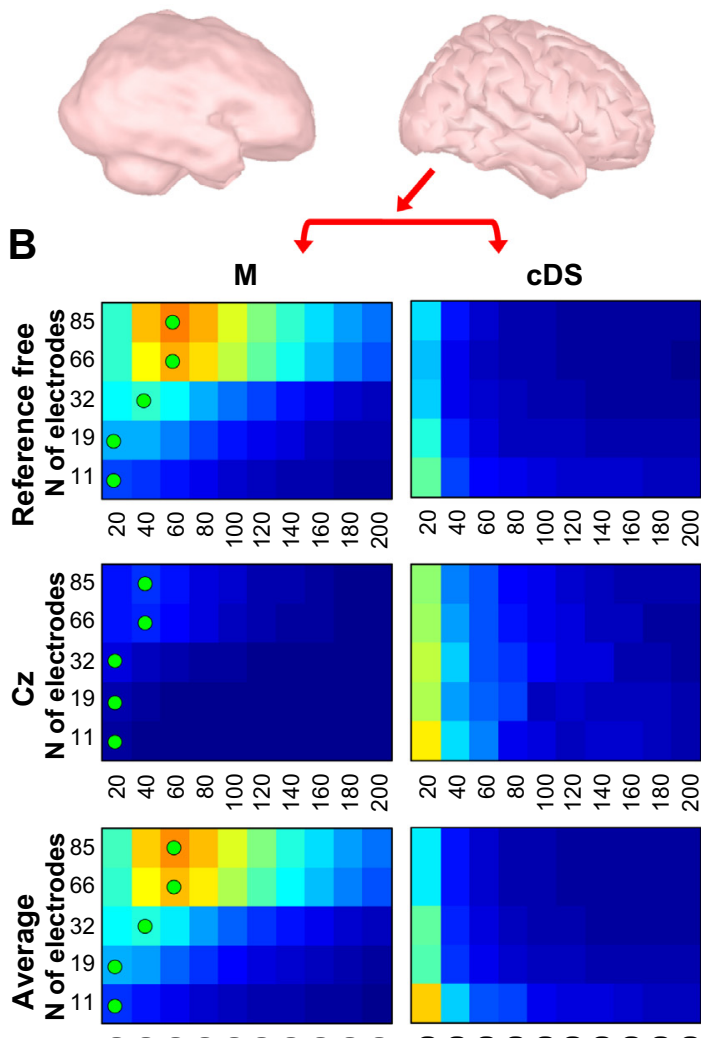

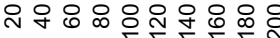
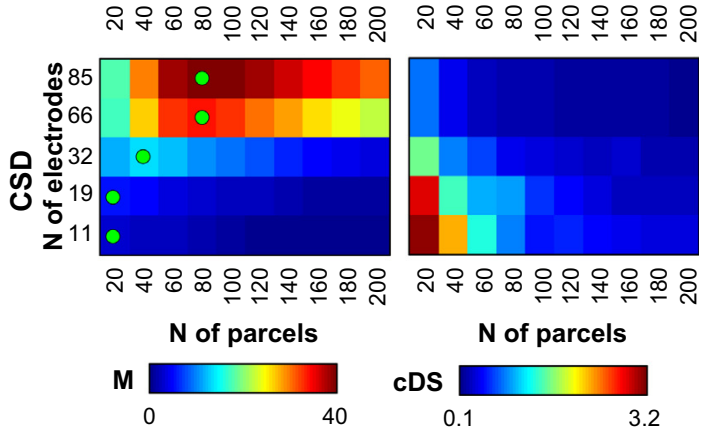

Fig. 7. Montage performance and coupling detection metrics for the gyrated model with skull conductivity $0.2 \mathrm{~S} / \mathrm{m}$. (A) Original baby brain model is shown on the left and gyrated brain model is shown on the right. (B) Maps for the number of discernible parcels $(M)$ are shown in the right column. The green circles depict the maximal $M$ values with each electrode number. Corresponding maps that were obtained with the original model are shown on Fig. 5A (right column). Maps for the cumulative degree of smearing $(c D S)$ are shown on the right. Corresponding maps for original model are shown on Fig. 6 (middle column). (For interpretation of the references to color in this figure legend, the reader is referred to the web version of this article.)

knowledge by showing that the considerably different dimensions and geometry of the neonatal head imply a need for specific neonatal head models in future studies on brain synchrony.

\subsection{Effects of increasing electrode numbers}

It is well established that the accuracy of EEG study can be improved by increasing spatial sampling with higher number of EEG electrodes (Lantz et al., 2003; Yamazaki et al., 2013). In support of this, we observed no 'ceiling effect' with respect to the electrode count (Figs. 5 and 6), which suggests that most of our metrics would continue to change with greater numbers of scalp electrodes than assessed here (Welch et al., 2014). Moreover, neonatal scalp 
A

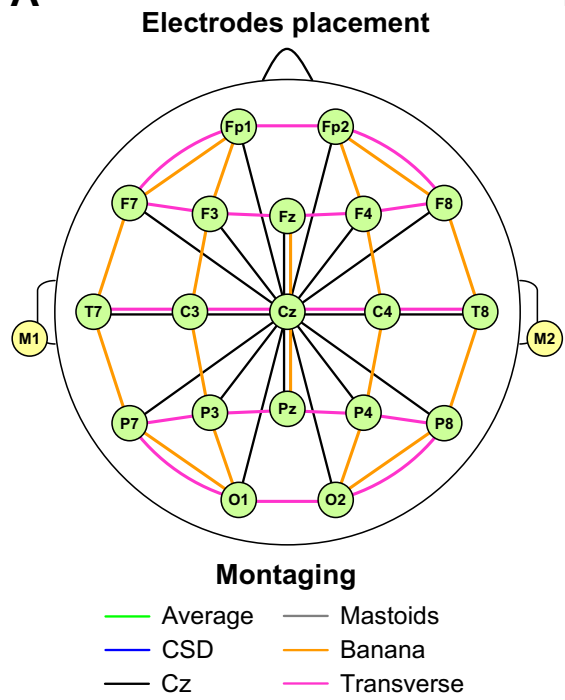

B
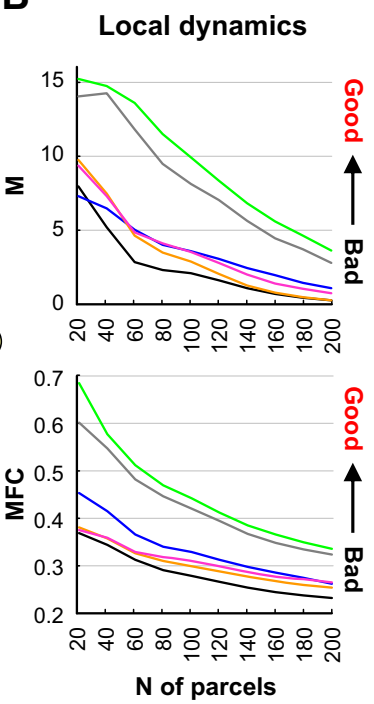

C
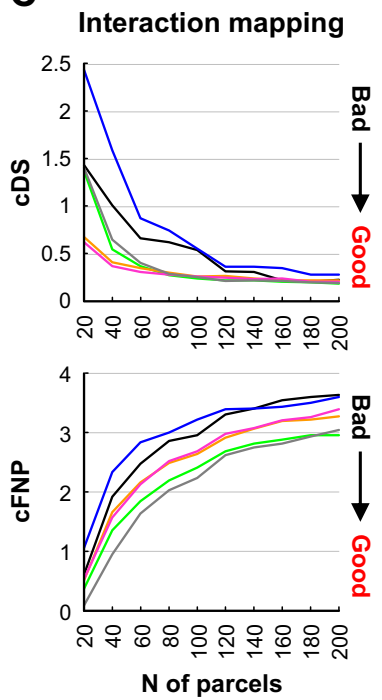

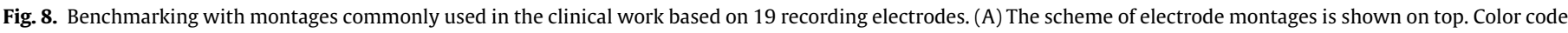

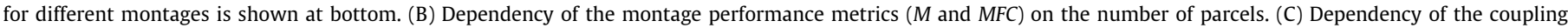

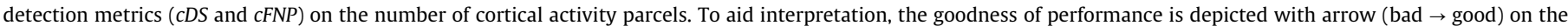
right side of the graphs.

EEG was recently shown to have very high spatial resolution, which implies that non-redundant information can be recorded with significantly denser spatial sampling than with the adult scalp EEG (Odabaee et al., 2013, 2014). However, analysis of synchrony from high number of signals is challenged by two types of confounders. First, higher number of sensors will lead to more smearing of true cortical synchronies between recorded electrodes, and needs attention in the methods used for spatial separation of signals or in computing synchrony (Palva and Palva, 2012, review). Second, increasing numbers of pair-wise synchrony estimates poses a statistical challenge due to multiple comparisons, and needs solutions akin to those used in neuroimaging (McIntosh and Mišic, 2013; Singh et al., 2011; Singh and Phillips, 2010). Recently introduced graph measures, especially those based on weighted and surrogate-normalized matrices (Boersma et al., 2011; Rubinov and Sporns, 2010), may effectively mitigate the challenge associated with massive pairwise comparisons and the underlying assumptions of temporal stationarity and inter-individual similarity (Sporns, 2013).

Our present study was designed to assess the ability of sensor-level scalp EEG to pick up correlation relationships in signals generated by numerous cortical parcels. An increase in parcel number is associated with a decrease in the parcel size (Fig. 2D), which, consequently, implies greater degree of signal mixing at scalp level. This mixing will set the upper limit to synchrony detection at scalp level, which is clearly seen as a 'ceiling effect' with respect to parcel number in our results (Fig. 5A). Both adult and neonatal EEG exhibit diminishing returns on increasing electrode count, but because of the greater conductivity of the neonatal skull, the relevant upper limit for electrode density in neonatal EEG is much higher than in adult EEG, and probably even higher than what is practically feasible to measure at present. To enhance spatial resolution beyond this level, one would need the combination of denser EEG sampling with source reconstruction methods to disentangle the source signals (Palva and Palva, 2012; Schoffelen and Gross, 2009).

\subsection{Effects of analysis montage}

Montage selection is one of the necessary steps in all EEG analysis, so we compared the different montages both quantitatively and qualitatively (Fig. 9). The conventional EEG analysis often relies on using variations of bipolar, linked mastoid reference or average reference montages, while the more recent, academically oriented studies (cf. Grieve et al., 2008; Omidvarnia et al., 2014; Roche-Labarbe et al., 2008; Tokariev et al., 2012) commonly rely on average and CSD montages. Our results show clearly that mixing signals results in a significant loss of specificity and sensitivity about spatial interactions, as in bipolar derivations, including common $C z$ reference. Our simulation did also show how the traditional mastoid reference may yield results that are comparable to average or CSD montage. In real life situation with human infants, however, use of mastoid reference is severely compromised if not fully precluded by the presence of frequent artefacts coming from movements, muscle artefacts (e.g. sucking) and cardiac artefacts. These considerations leave average and CSD montages only for studying brain interactions when one wishes to optimize both sensitivity and specificity. Our present findings slightly favored using average reference, which is also conceptually straightforward and simple to generate. Its practical utility is, however, compromised by higher amplitude artefacts in any of the signals. The practical advantages of CSD montage, in turn, include local specificity that reduces 'computational spread' of artefacts (e.g. muscle or movement) that occur in one or few channels only (Tenke and Kayser, 2012), as well as the possibility to interpolate bad channels. However, CSD becomes unreliable with lower electrode numbers because of its sensitivity to edge effects (Hjorth, 1975; Mackay, 1983), and CSD does also require operator input to define the parameters related to flexibility in spatial contours of scalp potential (Tenke and Kayser, 2012). These parameters have not been systematically studied in infants, but our present simulation results and recent empiric findings (Odabaee et al., 2013) suggest unsmoothed CSD to give the most reliable estimates. An alternative solution, reference electrode standardization technique (REST; Yao, 2001), has been developed to avoid the issues with reference electrodes, and it might provide a practical, comparable solution that merits further assessment.

\subsection{Spatial blurring of scalp EEG signals due to positioning inaccuracy}

An interesting, though indirect implication of our present findings is the confounder that arises from the narrow cortical 'field of 
19 electrodes

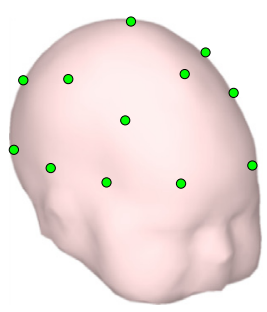

$\checkmark$

Local dynamics
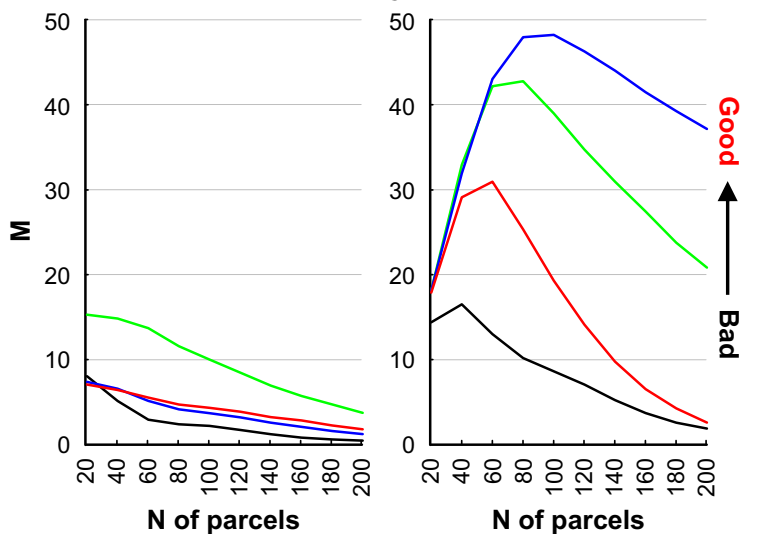

Interaction mapping

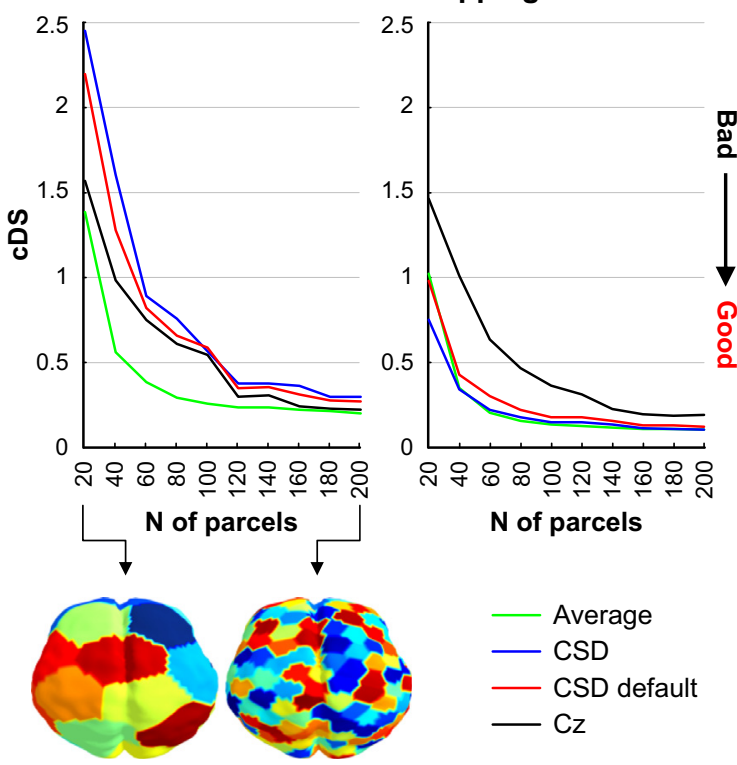

Fig. 9. Summary of the study findings in the context of 19 channel vs. 85 channel recordings. This illustrates how the findings of the present study can be used to aid analysis of datasets that are recorded with the standard (19 channel) or hdEEG (85 channel) settings. The upper graphs compare the ability of different montages (color code is shown in the bottom right corner of the figure) to detect local dynamics as estimated with $M$. Note the dramatically increased number of discernible cortical parcels $(M)$ with higher number of recording electrodes. The lower graphs compare the amount of spatial smearing with each montage. Note how spatial smearing is clearly lower with higher electrode count, however there are also clear differences between the montages. These graphs are generated from the more extensive analysis shown in the right column of Fig. 5A $(M)$ and the middle column of Fig. $6(c D S)$ where cases shown on this figure are highlighted with corresponding colors on 2D maps. Taken altogether, the results suggest that average reference is generally better with low number of recording electrodes while CSD montage becomes better when the recorded electrode count is higher. view' of scalp electrodes combined to their localization inaccuracy. Due to the neonatal head dimensions and high skull conductivity, each scalp EEG electrode mainly sees just a few centimeters of cortical activity. Using anatomical landmarks leads to a centimeter level inaccuracy in electrode positioning relative to the functional cortical areas (Hellstrom et al., 1963; Kabdebon et al., 2014). This implies considerable spatial variability in the relationships between standard electrode positions and functional cortical loci between different subjects, or between different recording sessions or gestational ages in the same subject. The practical outcome of this consideration is that, for instance, synchrony estimate between central ( $\mathrm{C} 3$ or $\mathrm{C} 4$ ) and parietal (P3 and P4) EEG signals may in one baby come from pre- and postcentral gyri, respectively, while in another baby they measure relationship between signals that are both coming from postcentral gyri. It is obvious that the brain interactions between these two signal pairs are different, resulting in poor comparability between such two EEG recordings. To overcome this challenge, we can envision two approaches. First, it is theoretically possible to improve spatial accuracy with very high electrode numbers, followed by spatially re-sampled EEG signals based on individualized MRI imaging. Second, it may often be more practical, especially in clinical populations, to study the network properties at more global level rather than to maximize spatial accuracy of signals themselves (Rubinov and Sporns, 2010). Recent studies have provided very useful tools to measure graph based network properties where spatial features may be extracted with post hoc, data-driven analysis of connectivity clusters. The latter approach has recently gained wide interest in neurophysiological studies of all age groups and in various neurocognitive disorders (Boersma et al., 2013; de Haan et al., 2009; Kim et al., 2013; Omidvarnia et al., 2014; Reijneveld et al., 2007).

\subsection{Limitations}

Our present study design includes multiple simplifications that do not qualitatively influence the overall conclusions but do affect the exact numerical values presented here: First, we used a BEM head model with a relatively smooth cortical source surface and mainly superficial, radial source orientations. The true brain source geometry is obviously more complex with both deeper sources and sulcal sources with tangential or variable orientations relative to the scalp surface. Sources in these areas would produce weaker signals with more complex scalp topographies, which would further increase the spatial mixing of source signals. These effects both limit the yield of sensor-level (scalp EEG) analyses and emphasize the need for source reconstruction approaches with realistic cortical models (Palva et al., 2013; Schoffelen and Gross, 2009). It is hence likely that the simplified source space used here overestimates to some extent the spatial resolution and the ability of any given number of electrodes to discern cortical activity. However, the attempts to generate realistically contoured cortical sources are significantly hampered by the lack of sufficiently reliable and accurate cortical segmentation of the neonate MRI, as well as by the so far poorly understood cortical activity mechanisms that in neonates are different from the adults (Brockmann et al., 2011; Colonnese and Khazipov, 2012; Kilb et al., 2011; Vanhatalo and Kaila, 2006). Second, our BEM model was only based on few tissue compartments, while recent studies in adults have shown added benefits from as many as eleven (Ramon et al., 2006) or 25 compartments (Irimia et al., 2013). Further segmentation of the neonatal MRI is, however, limited by the dimensions of each tissue type relative to the size of MRI voxels. Our present work showed that the total thickness of skull tissue in the neonate 
was often below $2 \mathrm{~mm}$, which makes its segmentation challenging with the currently available $0.9 \mathrm{~mm}$ voxel resolution. Third, the present study only assessed pair-wise synchronies although cortical activity in vivo always involves phase correlations among numerous cortical areas (Bressler and Menon, 2010; Palva et al., 2013). Such a realistic multivariate synchrony cannot, however, be addressed here directly, because there is no way of rigorously dissociating true from false positives in scalp EEG sensor-sensor correlations. Such work would require source modeling to have the truth matrix and the recorded matrix with the same axes (Korhonen et al., 2014).

\subsection{Future directions}

The overarching methodological implication from our study is that there are no unified optima in methodology for studying functional brain synchrony in neonates. For purely scientific purposes, it is easy to envisage near future increases in the use of high density EEG methods, however most studies on neonates remain driven by clinical questions that include recordings from sick patients. Such recording are usually carried out in the neonatal intensive care environment, and the EEG devices available for such a place will likely be technically suboptimal regarding their spatial sampling. Moreover, neonatal subjects in many disease groups are so rare, that studies on their pathophysiology will need to exploit clinical archives consisting of technically suboptimal recordings, in addition to limited prospective data collection with better recording settings.

The multidirectional effects between recording and analysis parameters studied in our work show that future work on brain synchrony can use either a bottom-up or a top-down approach: A bottom-up approach will first need to assume the hypothetical size of interacting cortical areas, which will define the needed number of electrodes, optimal analysis montage, as well as the associated sensitivity and specificity of synchrony estimates. A top-down approach will start from the given set of recording electrodes, which may be dictated by practical constraints (e.g. use of historical datasets), and that in turn dictates the analysis montages as well as defines the practical limits of detectable spatial accuracy in cortical synchrony.

\section{Acknowledgments}

This work was supported financially by Helsinki University Hospital (Finland), Sigrid Jusélius Foundation (Finland), Finnish Cultural Foundation (Finland), the Academy of Finland (Finland) grant 253130, Päivikki and Sakari Sohlberg Foundation (Finland), as well as Gyllenberg Foundation (Finland).

Conflict of interest: None of the authors have potential conflicts of interest to be disclosed.

\section{Appendix A. Supplementary data}

Supplementary data associated with this article can be found, in the online version, at http://dx.doi.org/10.1016/j.clinph.2015.04. 291.

\section{References}

André M, Lamblin MD, d'Allest AM, Curzi-Dascalova L, Moussalli-Salefranque F, S Nguyen The T, et al. Electroencephalography in premature and full-term infants. Developmental features and glossary. Clin Neurophysiol 2010;40:59-124.

Aydore S, Pantazis D, Leahy RM. A note on the phase locking value and its properties. Neuroimage 2013:74:231-44.

Baumgartner R, Ryner L, Richter W, Summers R, Jarmasz M, Somorjai R. Comparison of two exploratory data analysis methods for fMRI: fuzzy clustering vs. principal component analysis. Magn Reson Imaging 2000;18:89-94.

Boersma M, Smit DJ, de Bie HM, Van Baal GC, Boomsma DI, De Geus EJ, et al. Network analysis of resting state EEG in the developing young brain: structure comes with maturation. Hum Brain Mapp 2011;32:413-25.
Boersma M, Smit DJ, Boomsma DI, De Geus EJ, Delemarre-van de Waal HA, Stam CJ. Growing trees in child brains: graph theoretical analysis of electroencephalography-derived minimum spanning tree in 5- and 7-year-old children reflects brain maturation. Brain Connect 2013;3:50-60.

Bressler SL, Menon V. Large-scale brain networks in cognition: emerging methods and principles. Trends Cogn Sci 2010;14:277-90.

Brockmann MD, Poschel B, Cichon N, Hanganu-Opatz IL. Coupled oscillations mediate directed interactions between prefrontal cortex and hippocampus of the neonatal rat. Neuron 2011;71:332-47.

Colonnese M, Khazipov R. Spontaneous activity in developing sensory circuits: implications for resting state fMRI. Neuroimage 2012;62:2212-21.

de Haan W, Pijnenburg YA, Strijers RL, van der Made Y, Van der Flier WM, Scheltens $P$, et al. Functional neural network analysis in frontotemporal dementia and Alzheimer's disease using EEG and graph theory. BMC Neurosci 2009;10:101.

Despotovic I, Cherian PJ, De VM, Hallez H, Deburchgraeve W, Govaert P, et al. Relationship of EEG sources of neonatal seizures to acute perinatal brain lesions seen on MRI: a pilot study. Hum Brain Mapp 2013;34:2402-17.

Essl M, Rappelsberger P. EEG coherence and reference signals: experimental results and mathematical explanations. Med Biol Eng Comput 1998;36:399-406.

Geselowitz DB. On bioelectric potentials in an inhomogeneous volume conductor Biophys J 1967;7:1-11.

Gramfort A, Papadopoulo T, Olivi E, Clerc M. OpenMEEG: opensource software for quasistatic bioelectromagnetics. Biomed Eng Online 2010;9:45.

Grieve PG, Emerson RG, Fifer WP, Isler JR, Stark RI. Spatial correlation of the infant and adult electroencephalogram. Clin Neurophysiol 2003;114:1594-608.

Grieve PG, Emerson RG, Isler JR, Stark RI. Quantitative analysis of spatial sampling error in the infant and adult electroencephalogram. Neuroimage $2004 ; 21: 1260-74$.

Grieve PG, Isler JR, Izraelit A, Peterson BS, Fifer WP, Myers MM, et al. EEG functional connectivity in term age extremely low birth weight infants. Clin Neurophysiol 2008;119:2712-20.

Hämäläinen MS, Ilmoniemi RJ. Interpreting magnetic fields of the brain: minimum norm estimates. Med Biol Eng Comput 1994;32:35-42.

Hanson SJ, Rebecchi R, Hanson C, Halchenko YO. Dense mode clustering in brain maps. Magn Reson Imaging 2007;25:1249-62.

Hellstrom B, Karlsson B, Mussbichler H. Electrode placement in EEG of infants and its anatomical relationship studied radiographically. Electroencephalogr Clin Neurophysiol 1963;15:115-7.

Hjorth B. An on-line transformation of EEG scalp potentials into orthogonal source derivations. Electroencephalogr Clin Neurophysiol 1975;39:526-30.

Holmes CJ, Hoge R, Collins L, Woods R, Toga AW, Evans AC. Enhancement of MR images using registration for signal averaging. J Comput Assist Tomogr $1998 ; 22: 324-33$.

Irimia A, Goh SY, Torgerson CM, Chambers MC, Kikinis R, Van Horn JD. Forward and inverse electroencephalographic modeling in health and in acute traumatic brain injury. Clin Neurophysiol 2013;124:2129-45.

Jenkinson M, Beckmann CF, Behrens TE, Woolrich MW, Smith SM. FSL. Neuroimage 2012;62:782-90.

Jervis BW, Nichols MJ, Johnson TE, Allen E, Hudson NR. A fundamental investigation of the composition of auditory evoked potentials. IEEE Trans Biomed Eng 1983;30:43-50.

Kabdebon C, Leroy F, Simmonet H, Perrot M, Dubois J, Dehaene-Lambertz G. Anatomical correlations of the international 10-20 sensor placement system in infants. Neuroimage 2014;99:342-56.

Kayser J, Tenke CE. Principal components analysis of Laplacian waveforms as a generic method for identifying ERP generator patterns: I. Evaluation with auditory oddball tasks. Clin Neurophysiol 2006a;117:348-68.

Kayser J, Tenke CE. Principal components analysis of Laplacian waveforms as a generic method for identifying ERP generator patterns: II. Adequacy of lowdensity estimates. Clin Neurophysiol 2006b;117:369-80.

Kilb W, Kirischuk S, Luhmann HJ. Electrical activity patterns and the functional maturation of the neocortex. Eur J Neurosci 2011;34:1677-86.

Kim DJ, Bolbecker AR, Howell J, Rass O, Sporns O, Hetrick WP, et al. Disturbed resting state EEG synchronization in bipolar disorder: a graph-theoretic analysis. Neuroimage Clin 2013;2:414-23.

Korhonen O, Palva S, Palva JM. Sparse weightings for collapsing inverse solutions to cortical parcellations optimize M/EEG source reconstruction accuracy. J Neurosci Methods 2014;226:147-60.

Krüger HS, Brockmann MD, Salamon J, Ittrich H, Hanganu-Opatz IL. Neonatal hippocampal lesion alters the functional maturation of the prefrontal cortex and the early cognitive development in pre-juvenile rats. Neurobiol Learn Mem 2012;97:470-81.

Kybic J, Clerc M, Abboud T, Faugeras O, Keriven R, Papadopoulo T. A common formalism for the integral formulations of the forward EEG problem. IEEE Trans Med Imaging 2005;24:12-28.

Lantz G, Grave de PR, Spinelli L, Seeck M, Michel CM. Epileptic source localization with high density EEG: how many electrodes are needed? Clin Neurophysiol 2003;114:63-9.

Lin FH, Belliveau JW, Dale AM, Hämäläinen MS. Distributed current estimates using cortical orientation constraints. Hum Brain Mapp 2006;27:1-13.

Mackay DM. On-line source-density computation with a minimum of electrodes. Electroencephalogr Clin Neurophysiol 1983;56:696-8.

McIntosh AR, Mišic B. Multivariate statistical analyses for neuroimaging data. Annu Rev Psychol 2013;64:499-525.

Nunez PL, Srinivasan R, Westdorp AF, Wijesinghe RS, Tucker DM, Silberstein RB, et al. EEG coherency. I: Statistics, reference electrode, volume conduction, 
Laplacians, cortical imaging, and interpretation at multiple scales. Electroencephalogr Clin Neurophysiol 1997;103:499-515.

Nunez PL, Silberstein RB, Shi Z, Carpenter MR, Srinivasan R, Tucker DM, et al. EEG coherency II: experimental comparisons of multiple measures. Clin Neurophysiol 1999;110:469-86.

Odabaee M, Freeman WJ, Colditz PB, Ramon C, Vanhatalo S. Spatial patterning of the neonatal EEG suggests a need for a high number of electrodes. Neuroimage 2013;68:229-35.

Odabaee M, Tokariev A, Layeghy S, Mesbah M, Colditz PB, Ramon C, et al. Neonata EEG at scalp is focal and implies high skull conductivity in realistic neonatal head models. Neuroimage 2014;96:73-80.

Omidvarnia A, Fransson P, Metsaranta M, Vanhatalo S. Functional bimodality in the brain networks of preterm and term human newborns. Cereb Cortex 2014;24:2657-68.

Palva S, Palva JM. Discovering oscillatory interaction networks with M/EEG: challenges and breakthroughs. Trends Cogn Sci 2012;16:219-30.

Palva JM, Zhigalov A, Hirvonen J, Korhonen O, Linkenkaer-Hansen K, Palva S. Neuronal long-range temporal correlations and avalanche dynamics are correlated with behavioral scaling laws. Proc Natl Acad Sci U S A 2013;110:3585-90.

Pascual-Marqui RD, Lehmann D. Topographic maps, source localization inference and the reference electrode: comments on a paper by Desmedt et al Electroencephalogr Clin Neurophysiol 1993;88:532-6.

Ramon C, Haueisen J, Schimpf PH. Influence of head models on neuromagnetic fields and inverse source localizations. Biomed Eng Online 2006;5:55.

Reijneveld JC, Ponten SC, Berendse HW, Stam CJ. The application of graph theoretical analysis to complex networks in the brain. Clin Neurophysio 2007; 118:2317-31.

Roche-Labarbe N, Aarabi A, Kongolo G, Gondry-Jouet C, Dumpelmann M, Grebe R et al. High-resolution electroencephalography and source localization in neonates. Hum Brain Mapp 2008;29:167-76.

Rubinov M, Sporns O. Complex network measures of brain connectivity: uses and interpretations. Neuroimage 2010;52:1059-69.

Ryynanen OR, Hyttinen JA, Laarne PH, Malmivuo JA. Effect of electrode density and measurement noise on the spatial resolution of cortical potential distribution. IEEE Trans Biomed Eng 2004;51:1547-54.

Ryynanen OR, Hyttinen JA, Malmivuo JA. Effect of measurement noise and electrode density on the spatial resolution of cortical potential distribution with different resistivity values for the skull. IEEE Trans Biomed Eng 2006;53:1851-8.

Scherg M, Ille N, Bornfleth H, Berg P. Advanced tools for digital EEG review: virtual source montages, whole-head mapping, correlation, and phase analysis. J Clin Neurophysiol 2002;19:91-112.

Schoffelen JM, Gross J. Source connectivity analysis with MEG and EEG. Hum Brain Mapp 2009;30:1857-65.

Singh AK, Phillips S. Hierarchical control of false discovery rate for phase locking measures of EEG synchrony. Neuroimage 2010;50:40-7.
Singh AK, Asoh H, Phillips S. Optimal detection of functional connectivity from highdimensional EEG synchrony data. Neuroimage 2011:58:148-56.

Smith SM, Jenkinson M, Woolrich MW, Beckmann CF, Behrens TE, Johansen-Berg H, et al. Advances in functional and structural MR image analysis and implementation as FSL. Neuroimage 2004;23(Suppl. 1):S208-19.

Sporns 0 . Network attributes for segregation and integration in the human brain. Curr Opin Neurobiol 2013;23:162-71.

Stam CJ, van Straaten EC. The organization of physiological brain networks. Clin Neurophysiol 2012;123:1067-87.

Tadel F, Baillet S, Mosher JC, Pantazis D, Leahy RM. Brainstorm: a user-friendly application for MEG/EEG analysis. Comput Intell Neurosci 2011;2011:879716.

Tenke CE, Kayser J. Generator localization by current source density (CSD): implications of volume conduction and field closure at intracranial and scalp resolutions. Clin Neurophysiol 2012;123:2328-45.

Tognoli E, Kelso JA. Brain coordination dynamics: true and false faces of phase synchrony and metastability. Prog Neurobiol 2009;87:31-40.

Tokariev A, Palmu K, Lano A, Metsäranta M, Vanhatalo S. Phase synchrony in the early preterm EEG: development of methods for estimating synchrony in both oscillations and events. Neuroimage 2012:60:1562-73.

Vanhatalo S, Kaila K. Development of neonatal EEG activity: from phenomenology to physiology. Semin Fetal Neonatal Med 2006;11:471-8.

Vinck M, Oostenveld R, van Wingerden M, Battaglia F, Pennartz CM. An improved index of phase-synchronization for electrophysiological data in the presence of volume-conduction, noise and sample-size bias. Neuroimage 2011;55:1548-65.

Wallois F, Patil A, Kongolo G, Goudjil S, Grebe R. Haemodynamic changes during seizure-like activity in a neonate: a simultaneous AC EEG-SPIR and highresolution DC EEG recording. Neurophysiol Clin 2009;39:217-27.

Welch MG, Myers MM, Grieve PG, Isler JR, Fifer WP, Sahni R, et al. Electroencephalographic activity of preterm infants is increased by Family Nurture Intervention: a randomized controlled trial in the NICU. Clin Neurophysiol 2014;125:675-84.

Wolpaw JR, Wood CC. Scalp distribution of human auditory evoked potentials. I. Evaluation of reference electrode sites. Electroencephalogr Clin Neurophysiol $1982 ; 54: 15-24$.

Yamazaki M, Tucker DM, Terrill M, Fujimoto A, Yamamoto T. Dense array EEG source estimation in neocortical epilepsy. Front Neurol 2013;4:42.

Yao D. A method to standardize a reference of scalp EEG recordings to a point at infinity. Physiol Meas 2001;22:693-711.

Yao D, Wang L, Oostenveld R, Nielsen KD, Arendt-Nielsen L, Chen AC. A comparative study of different references for EEG spectral mapping: the issue of the neutral reference and the use of the infinity reference. Physiol Meas 2005;26:173-84.

Yao D, Wang L, Arendt-Nielsen L, Chen AC. The effect of reference choices on the spatio-temporal analysis of brain evoked potentials: the use of infinite reference. Comput Biol Med 2007;37:1529-38. 